\title{
Amendment of the Japanese Consensus Guidelines \\ for Autoimmune Pancreatitis, 2013 \\ I. Concept and diagnosis of autoimmune pancreatitis
}

\author{
Kazuichi Okazaki • Shigeyuki Kawa • Terumi Kamisawa • Tetsuhide Ito • \\ Kazuo Inui $\cdot$ Hiroyuki Irie $\cdot$ Takayoshi Nishino $\cdot$ Kenji Notohara $•$ \\ Isao Nishimori $\cdot$ Shigeki Tanaka $\cdot$ Toshimasa Nishiyama $\cdot$ Koichi Suda $\cdot$ \\ Keiko Shiratori - Masao Tanaka - Tooru Shimosegawa - The Working Committee of the Japan Pancreas Society \\ and the Research Committee for Intractable Pancreatic Disease supported by the Ministry of Health, Labour and \\ Welfare of Japan
}

Received: 27 January 2014/ Accepted: 2 February 2014/Published online: 18 March 2014

(C) Springer Japan 2014

\begin{abstract}
Background In response to the proposal of the international consensus diagnostic criteria (ICDC) for autoimmune pancreatitis (AIP) and the Japanese diagnostic criteria in 2011, the 2009 Japanese consensus guidelines for managing AIP required revision.

Methods Three committees [the professional committee for making clinical questions (CQs) and statements by Japanese specialists, the expert panelist committee for rating statements by the modified Delphi method, and the evaluating committee by moderators] were organized. Fifteen specialists for AIP extracted the specific clinical
\end{abstract}

This article is the first of a three-article series on the Japanese consensus guidelines. The members of the Working Committee are listed in the "Appendix 2" in the text.

\section{K. Okazaki ( $($ )}

Department of Gastroenterology and Hepatology, Kansai

Medical University, 2-3-1 Shinmachi, Hirakata,

Osaka 573-1191, Japan

e-mail: okazaki@hirakata.kmu.ac.jp

S. Kawa

Center for Health, Safety and Environmental Management,

Shinshu University, Matsumoto, Japan

T. Kamisawa

Department of Internal Medicine, Tokyo Metropolitan

Komagome Hospital, Tokyo, Japan

T. Ito

Department of Medicine and Bioregulatory Science, Graduate

School of Medical Sciences, Kyushu University, Fukuoka, Japan

K. Inui

Department of Gastroenterology, Second Teaching Hospital,

Fujita Health University, Nagoya, Japan statements from 1,843 articles published between 1963 and 2012 (obtained from Pub Med and a secondary database, and developed the CQs and statements. The expert panel individually rated the clinical statements using a modified Delphi approach, in which a clinical statement receiving a median score greater than seven on a nine-point scale from the panel was regarded as valid.

Results The professional committee created $13 \mathrm{CQs}$ and statements for the current concept and diagnosis of AIP, 6 for extra-pancreatic lesions, 6 for differential diagnosis, and 11 for treatment.

Conclusion After evaluation by the moderators, amendments to the Japanese consensus guidelines for AIP have been proposed for 2013 .

H. Irie

Department of Radiology, Faculty of Medicine, Saga University,

Saga, Japan

T. Nishino

Department of Gastroenterology, Tokyo Women's Medical

University, Yachiyo Medical Center, Yachiyo, Japan

K. Notohara

Department of Anatomic Pathology, Kurashiki Central Hospital, Kurashiki, Okayama, Japan

I. Nishimori

Nishimori Clinic, Kochi, Japan

S. Tanaka

Department of Acupuncture and Moxibusion, Tokyo Ariake

University of Medical and Health Sciences, Tokyo, Japan 
Keywords Autoimmune pancreatitis - Guideline Diagnosis · Treatment · Delphi method

\section{Introduction}

Since Yoshida et al. [1], first proposed the concept of autoimmune pancreatitis (AIP) in 1995, AIP has been accepted worldwide as a distinctive type of pancreatitis [16]. Due to the increasing numbers of cases, several issues in the management of AIP were raised in Japan. These issues are (1) diagnosis and management of atypical or indeterminate AIP, (2) differentiation from pancreas cancer, (3) evaluation of OOIs, (4) diagnosis and treatment of recurrent cases, and (5) different diagnostic criteria in Japan and other countries [4, 5]. To resolve these issues, the Japan Pancreas Society (JPS) and the Research Committee for Intractable Pancreatic Disease supported by the Ministry of Health, Labour and Welfare of Japan (RCIPD-MHLWJ), proposed the Japanese consensus guidelines for the management of AIP in 2009 [6]. In 2011, the International Consensus Diagnostic Criteria for AIP (ICDC) [9] were proposed. The ICDC proposed two subtypes, type 1 AIP, which is associated with IgG4, and type 2 AIP, which is associated with granulocytic epithelial lesion (GEL). Lymphoplasmacytic sclerosing pancreatitis (LPSP) is a pancreatic manifestation of IgG4-related disease (IgG4-RD) characterized by increased serum IgG4 and abundant infiltration of IgG4positive plasmacytes, obliterative phlebitis and storiform fibrosis. In Japan, LPSP is more often observed, whereas idiopathic duct-centric chronic pancreatitis (IDCP) characterized by GEL is rare [4-8]. One of the major differences between the 2002 and 2006 Japanese criteria and the ICDC is in the therapeutic use of steroids. The previous Japanese criteria [3-5] did not recommend facile therapeutic use of steroids. The revised version of the JPS criteria (JPS-2011)

\section{T. Nishiyama}

Department of Public Health and Hygiene, Kansai Medical University, Osaka, Japan

K. Suda

Department of Pathology, Tokyo-West Tokushukai Hospital, Tokyo, Japan

\section{K. Shiratori}

Department of Gastroenterology, Tokyo Women's Medical University, Tokyo, Japan

M. Tanaka

Department of Surgery and Oncology, Graduate School of Medical Sciences, Kyushu University, Fukuoka, Japan

T. Shimosegawa

Division of Gastroenterology, Tohoku University Graduate

School of Medicine, Sendai, Japan for type 1 AIP $[10,11]$ was proposed in response to the ICDC's inclusion of response to steroid treatment. The number of publications on AIP increased from 871 to 1,843 between 2008 and 2012 (in the PubMed database). In light of this additional research, the Japanese consensus guidelines need to be revised. Most of the evidence levels of the specific clinical statements and a secondary database were still lower than grade III as proposed by the Agency for Health Care Policy and Research in 1993. Therefore, we have developed the revised version of the consensus guidelines using the modified Delphi approach [5-8, 12]. Briefly, to establish consensus, three committees (the professional committee for making clinical questions and statements by Japanese specialists for AIP, the expert panel committee for rating statements using the modified Delphi method, and the evaluating committee comprised of moderators) were organized. During the first phase, 15 specialists (11 pancreatologists, 2 radiologists, 1 respiratory system expert and 1 pathologist) were selected from the members of the RCIPD-MHLWJ. These specialists revised the 36 clinical questions (CQs) and statements for (1) concept and diagnosis (13 CQs), (2) extra-pancreatic lesions (6 CQs), (3) differential diagnosis (6 CQs) and (4) treatment (11 CQs) based on the selected papers [6-8], which focus on the concept and diagnosis CQs.

The expert panelists (ten pancreatologists) individually rated the clinical statements for appropriateness, and discussed areas of disagreement and uncertainty [5-8, 12]. Ratings of appropriate methods for the management of AIP were developed using a modified Delphi approach. Rating was on a nine-point scale, with one being highly inappropriate and nine being highly appropriate. A clinical statement receiving a median score greater than seven was regarded as valid. The specialists revised some of the clinical statements after discussion with expert panelists, and then the revised clinical statements were rated again. Based on the two-round modified Delphi approach, guideline statements for diagnosis and management of AIP were developed. In addition to the specialist and expert panels, the moderators included one pancreatologist, one surgeon, one pathologist and one internist, each of whom were also familiar with epidemiology and the modified Delphi approach [5-8]. The moderators reviewed the literature, collected clinical statements from the literature as well as from a survey of the professionals, facilitated the panelist meetings, and analyzed the data. Because available clinical evidence regarding the diagnosis and management of AIP is limited, we could not set a suitable recommendation level for some clinical statements. In the revised consensus-based guidelines, the statements for clinical practice were evaluated as "strongly recommendable" (level A) or "strongly unrecommendable (level D)" for receiving a score of nine, and "ordinarily recommendable" (level B), "unrecommendable" (level C), or 
"conflicting benefits and harms" (level I) for that less than nine according to the grading proposed by United States Preventive Services Task Force [13].

\section{Clinical questions and statements}

\section{Concept and Diagnosis}

\section{CQ-I-1. What is "autoimmune pancreatitis (AIP)"?}

- AIP is a distinct form of pancreatitis clinically characterized by frequent presentation with obstructive jaundice with or without a pancreatic mass, histologically by a lymphoplasmacytic infiltrate and fibrosis and therapeutically by a dramatic response to steroids.

- AIP is classified as two subtypes, type 1 and type 2 . Type 1 AIP is more prevalent in Japan; references to AIP in Japanese literature usually mean type 1 AIP.

- Type 1 AIP is lymphoplasmacytic sclerosing pancreatitis (LPSP) characterized by massive infiltration of lymphocytes and plasmacytes, especially IgG4-positive plasmacyte; storiform fibrosis; and obliterative phlebitis. It is a pancreatic manifestation of a systemic disorder, IgG4-related disease (IgG4-RD).

- Type 2 AIP (also called IDCP) or AIP with GEL, is more commonly observed in Europe and the United States. Type 2 AIP exhibits neutrophilic lesions and, therefore, is a different condition from type 1 AIP.

Description AIP is a distinct form of pancreatitis characterized clinically by frequent presentation with obstructive jaundice with or without a pancreatic mass, histologically by a lymphoplasmacytic infiltrate and fibrosis and therapeutically by a dramatic response to steroids.

The original concept of AIP was proposed in Japan [1] and was defined as a pancreatitis whose pathogenesis could possibly involve autoimmune mechanisms [1-8]. Autoimmune mechanisms were suspected due to characteristic findings, such as hypergammaglobulinemia, increased serum levels of $\mathrm{IgG}$ or $\mathrm{IgG} 4$, presence of autoantibodies, and effective response to steroid therapy. Patients with AIP occasionally exhibit other organ involvement (OOI) such as sclerosing cholangitis, sclerosing sialadenitis, retroperitoneal fibrosis, enlarged coeliac and hilar lymph nodes, chronic thyroiditis, or interstitial nephritis. All of them show similar pathological findings with abundant infiltration of IgG4-positive cells as well as high serum IgG4, which support the possibility that AIP is a systemic disorder associated with pancreatic lesions. After several proposals of nomenclatures such as the IgG4-related systemic sclerosing disease [14], systemic IgG4-related plasmacytic syndrome (SIPS) [15] and IgG4-positive multi-organ lymphoproliferative syndrome (IgG4 MOLPS), [16] the consensus nomenclature of IgG4-related disease (IgG4-RD) [17-20] was proposed. Therefore, AIP related to $\mathrm{IgG} 4$ is now regarded as a pancreatic manifestation of IgG4-RD. Histopathological findings show lymphoplasmacytic sclerosing pancreatitis (LPSP) [21] characterized by (1) massive infiltration of lymphocytes and plasmacytes, (2) especially IgG4-positive plasmacytes more than 10 cells/high power field $(400 \times)$, (3) storiform fibrosis, and (4) obliterative phlebitis. It is commonly seen in elderly males, and is comparable to lymphoplasmacytic sclerosing pancreatitis (LPSP), which is characterized by histopathological findings of pronounced infiltration of lymphocytes and plasmacytes, infiltration of IgG4-positive plasmacytes, storiform fibrosis, and obstructive phlebitis.

Cases associated with ulcerative colitis in young patients, mainly reported in Europe and the US, show typical pathological neutrophilic lesions called IDCP [22] or AIP with GEL $[23,24]$. In addition to histopathological findings, no hematological markers suggest that their pathological conditions are different from LPSP [11]. Although typical pancreatic images in both LPSP and IDCP show diffuse swelling of more than one-third of the pancreas, some atypical and indeterminate cases of segmental/focal swelling or mass-forming type [9-11] are necessary to be differentiated from pancreatic cancer. Based on these findings, the recent international consensus diagnostic criteria (ICDC) for AIP [9] proposed the current concept of AIP and classification as two subtypes, type 1 (LPSP) and type 2 AIP (IDCP). As most of AIP are type 1 AIP in Japan [10, 11], use of the simple term of AIP usually means type 1 AIP in the present guidelines (Table 1).

\section{CQ-I-2. Are there characteristic clinical symptoms in AIP?}

- There are no specific symptoms seen in patients with AIP. However, in many cases, patients with type 1 AIP

Table 1 Recommendation levels based on consensus

Level A Recommendation that procedure or treatment is useful or effective

Level B Recommendation in favor of procedure or treatment being useful or effective

Level C Recommendation's usefulness or efficacy less well established

Level D Recommendation that procedure or treatment is not useful or effective but may be harmful

Level I The balance of benefits and harms cannot be determined, because evidence is lacking, of poor quality, or conflicting

http://www.ahrq.gov/clinic/3rduspstf/ratings.htm 
show; obstructive jaundice; symptoms of diabetes mellitus; accompanying extra-pancreatic lesions, and minor to no abdominal pain. Those with type 2 AIP commonly have abdominal pain and acute pancreatitis.

Description Patients with type 1 AIP do not show the type of severe abdominal pain seen in those with acute pancreatitis or with acute exacerbation of chronic pancreatitis. Abdominal pain is mild to nonexistent, $[5,19,25-$ 28] although there have been a few cases reported where the disease started as acute pancreatitis or severe pancreatitis [29, 30]. One-third to one-half of patients show obstructive jaundice or mild abdominal pain, and 15 percent have shown back pain or weight loss [31, 32]. More than half of cases are associated with sclerosing cholangitis, diabetes mellitus, sclerosing sialoadenitis/dacryoadenitis, or retroperitoneal fibrosis, showing obstructive jaundice, polydipsia,/polyuria or malaise, xerostomia/ xerophthalmia, or hydronephrosis, respectively [31]. Those with type 2 AIP commonly have abdominal pain and acute pancreatitis [9] (Table 2).

\section{CQ-I-3. How is AIP found?}

- In many cases, patients go to see doctors with complaints such as minor abdominal pain, general malaise, jaundice, or dry mouth (Level of recommendation: B).

- In many cases, AIP is found when patients who have increased levels of biliary enzymes, obstructive jaundice, or diabetes mellitus are tested for pancreatic or biliary duct cancers in a differential diagnosis (Level of recommendation: B).

- In many cases, an enlarged pancreas demonstrated by abdominal ultrasonography leads to the detection of AIP (Level of recommendation: B).

Description In more than half of AIP cases, patients visit the hospital for symptoms such as minor abdominal pain, general malaise, jaundice, or dry mouth [1, 25-32]. A urine test or general blood biochemical test shows abnormal levels of pancreatic or biliary enzymes. In other cases, an

Table 2 Clinical symptoms in AIP

\begin{tabular}{ll}
\hline Obstructive jaundice & $33-59 \%$ \\
Abdominal pain & $32 \%$ \\
Back pain & $15 \%$ \\
Body weight loss & $15 \%$ \\
Anorexia & $9 \%$ \\
General fatigue & $9 \%$ \\
Abnormal stool & $7 \%$ \\
Fever & $6 \%$ \\
No symptoms & $15 \%$ \\
\hline
\end{tabular}

increased level of CA19-9 is observed; pancreatic imaging tests such as abdominal ultrasound, CT or MRI show a diffusely or locally enlarged pancreas, or a pancreatic mass may also be found. In many cases the disease is found in the course of a differential diagnosis against pancreatic or biliary cancers $[1,6-11,25-32]$. AIP is also found during the close examination of extra-pancreatic lesions; e.g., during the differential diagnosis against primary sclerosing cholangitis (PSC); in examination of suspected Sjögren's syndrome by a head/neck-otolaryngologist, ophthalmologist, or collagen disease-rheumatologist; or in examination for retroperitoneal fibrosis by an urologist. The rate of association with other autoimmune diseases is not clear. There have been reports, mainly in Europe and the United States, of cases associated with juvenile ulcerative colitis showing evidence of IDCP [9, 22] or GEL [23, 24]. Conversely, cases associated with ulcerative colitis or primary biliary cirrhosis are rarely seen in Japan [6-8].

\section{CQ-I-4. What are the characteristic blood-biochemical and immunological findings in AIP?}

- Although there are no disease-specific serum biochemical findings, increased serum levels of pancreatic enzymes, biliary enzymes and total bilirubin are commonly observed in AIP (Level of recommendation: A).

- Serum levels of IgG4 have the highest diagnostic value as a single serological diagnostic method among all the available ones; however, it is not disease specific (Level of recommendation: A).

- High serum IgG or the presence of non-specific antibodies such as antinuclear antibodies or rheumatoid factor suggest the possibility of AIP (Level of recommendation: B).

Description Most cases of AIP are discovered when patients show increased levels of biliary enzymes, obstructive jaundice, or diabetes mellitus, which are usually reflected in biochemical tests. Abnormal biliary findings are seen in many cases; $60-82 \%$ of cases exhibit an increase of biliary enzymes: and 39-62 \% of cases exhibit an increase of total bilirubin [32-35]. Compared to cases of acute pancreatitis or acute exacerbation of chronic pancreatitis, the occurrence rate of abnormal levels of serum pancreatic enzymes is lower, $36-64 \%$ [32, 33], and the levels rarely become abnormally high. There have been reports of increased levels of peripheral eosinophil granulocytes [32] and activated T-lymphocytes (CD4-positive, CD8-positive) [33].

Immunological examinations show high incidences of hypergammaglobulinemia (43\%), increased levels of serum IgG $(62-80 \%)$, increased levels of serum IgG4 (68-92\%) [32-35] ), antinuclear antibodies (40-64\%), rheumatoid factor $(25 \%)[32,33]$ and Th2 predominance 
over Th1 in the local lesions [36, 37]. However, these results are not disease-specific. Some reports have shown the presence of autoantibodies, such as anti-carbonic anhydrase II antibodies (55\%) or anti-lactoferrin antibodies $(75 \%)$ in patients with AIP, although they generally cannot be tested $[32,33]$. Anti-SSA/B antibodies or antimitochondrial antibodies, on the other hand, are rarely seen [32, 33]. Among all serological diagnostic methods, an increased level of serum IgG4 has the highest diagnostic value as a single method because of its sensitivity ( $80 \%)$ and its specificity (98\%) in differentiating AIP from pancreatic cancer; however, it is not disease specific. The sensitivity and specificity of serum IgG are 70 and $75 \%$, respectively, and the positive ratios of antinuclear antibodies and rheumatoid factor are 60 and $20-30 \%$, respectively. Even when $\operatorname{IgG}$ is combined with antinuclear antibodies or rheumatoid factor, the sensitivity is $91 \%$ but the specificity is $61 \%$; the specificity is lower than that for $\mathrm{IgG} 4$, although the sensitivity is equivalent to that for IgG4 [34]. (Refer to CQ-III-2).

\section{CQ-I-5. Are there pancreatic exocrine and endocrine dysfunctions?}

- AIP is often associated with pancreatic exocrine and endocrine dysfunctions (e.g., diabetes mellitus). Occurrence ratios are about 80 and $70 \%$ for exocrine and endocrine dysfunctions, respectively, (Level of recommendation: A).

Description AIP is in many cases associated with pancreatic exocrine and endocrine dysfunction (e.g., diabetes mellitus.) According to the fact-finding survey conducted in 2000 by the Ministry of Health and Welfare Investigation Research Team for Special Intractable Pancreas Disease, $80.6 \%$ of the cases studied showed abnormal pancreatic exocrine function [in which the abnormality is defined as $70 \%$ or lower secretion in the BT-PABA (PFD test)], and $70.0 \%$ of the cases showed exocrine dysfunction (as determined by the secretin test), comparable to that of confirmed cases of chronic pancreatitis. Additionally, $77.0 \%$ of the cases were reported to be associated with diabetes mellitus [31]. Studies by individual medical facilities have reported that $83-88 \%$ of the cases were associated with secretion dysfunction, and $42-78 \%$ with diabetes mellitus [38-40]. The diabetes mellitus accompanying AIP was analyzed in detail in a national factfinding survey conducted in 2006 [35]. Among those AIP patients who sought medical treatment in 2002, $66.5 \%$ of cases were found to have associated diabetes mellitus; Of those, $33.3 \%$ had diabetes mellitus prior to the onset of AIP, and $51.6 \%$ started developing diabetes mellitus around the same time as the onset of pancreatitis. Among those patients having diabetes mellitus, $14 \%$ developed diabetes after steroid treatment [35], suggesting that such diabetes may be caused by long-term steroid treatment. There are some cases where pancreatic endocrine dysfunction was improved by steroid treatment; however, since not all cases improved, it can be stated that medical conditions that have progressed far enough to cause some degree of organic change can not be reversed. (Refer to CQ-IV-9.)

In AIP, the mechanism of pathogenesis of pancreatic exocrine dysfunction is assumed to involve the following: decreased secretion of pancreatic enzymes associated with collapsed acinar cells caused by pronounced cellular infiltration mainly of plasmacytes and fibrosis; and obstructed flow of pancreatic juice due to inflammatory cell infiltration around the pancreatic ducts and subsequent narrowing of pancreatic ducts [35, 39-41]. A recent study suggested that mislocalization of the cystic fibrosis transmembrane conductance regulator (CFTR), which plays a central role in pancreatic duct $\mathrm{HCO}_{3}{ }^{-}$secretion, and upregulation of aquaporin-1 (AQP1) on the plasma membrane and in the cytoplasm of pancreatic duct cells may be involved in the development of AIP [42]. Corticosteroids reduce inflammation and restore both digestive enzyme and $\mathrm{HCO}_{3}{ }^{-}$secretion in patients with AIP by regenerating acinar cells and correcting CFTR localization in pancreatic duct cells [42]. In contrast, the mechanism of pathogenesis of diabetes mellitus is assumed to be affected by both obstructed blood flow of endocrine glands (islets of Langerhans) associated with the fibrosis of exocrine glands, and damaged islets of Langerhans due to the spreading of inflammation [40, 41].

CQ-I-6. What are the characteristic findings of abdominal ultrasonography in AIP ?

- Abdominal ultrasonography is effective for the diagnosis of AIP. Ultrasonic findings in patients with AIP are characterized by a diffusely or locally enlarged pancreas with low echo; A diffusely enlarged pancreas is called a "sausage-like" pancreas. (Level of recommendation: A).

Description The Japanese Clinical Diagnostic Criteria of Autoimmune Pancreatitis [3-5, 10, 11] states that a "diffusely or locally enlarged pancreas is detected by an abdominal ultrasound test, an abdominal X-ray test, or an abdominal MRI test." Ultrasonography is the initial clinical test performed, and serves as a tool to diagnose AIP. However, in some cases, patients are found to have AIP during physical examinations [43].

A diffusely enlarged pancreas appears as a low-echo area in general (Fig. 1a) and has a so-called "sausage-like" appearance [44]. No dilatation of the main pancreatic duct 
Fig. 1 Abdominal ultrasonography in AIP. a Diffuse type: a diffusely enlarged pancreas appears as a low-echo area with high echoic spots and has a so-called "sausage-like" appearance. b Tumor forming type: The main duct (arrows) is found to penetrate through the mass (duct-penetrating sign) in the case of a locally enlarged pancreas with a clear margin. c Multiple mass forming type: low echoic masses are observed in the pancreas head and body (arrows) and normal parenchyma is seen in the interstitial segment of these masses. d IgG4-related sclerosing cholangitis: moderately homogenous lowechoic wall thickness is observed in the upper and middle bile duct
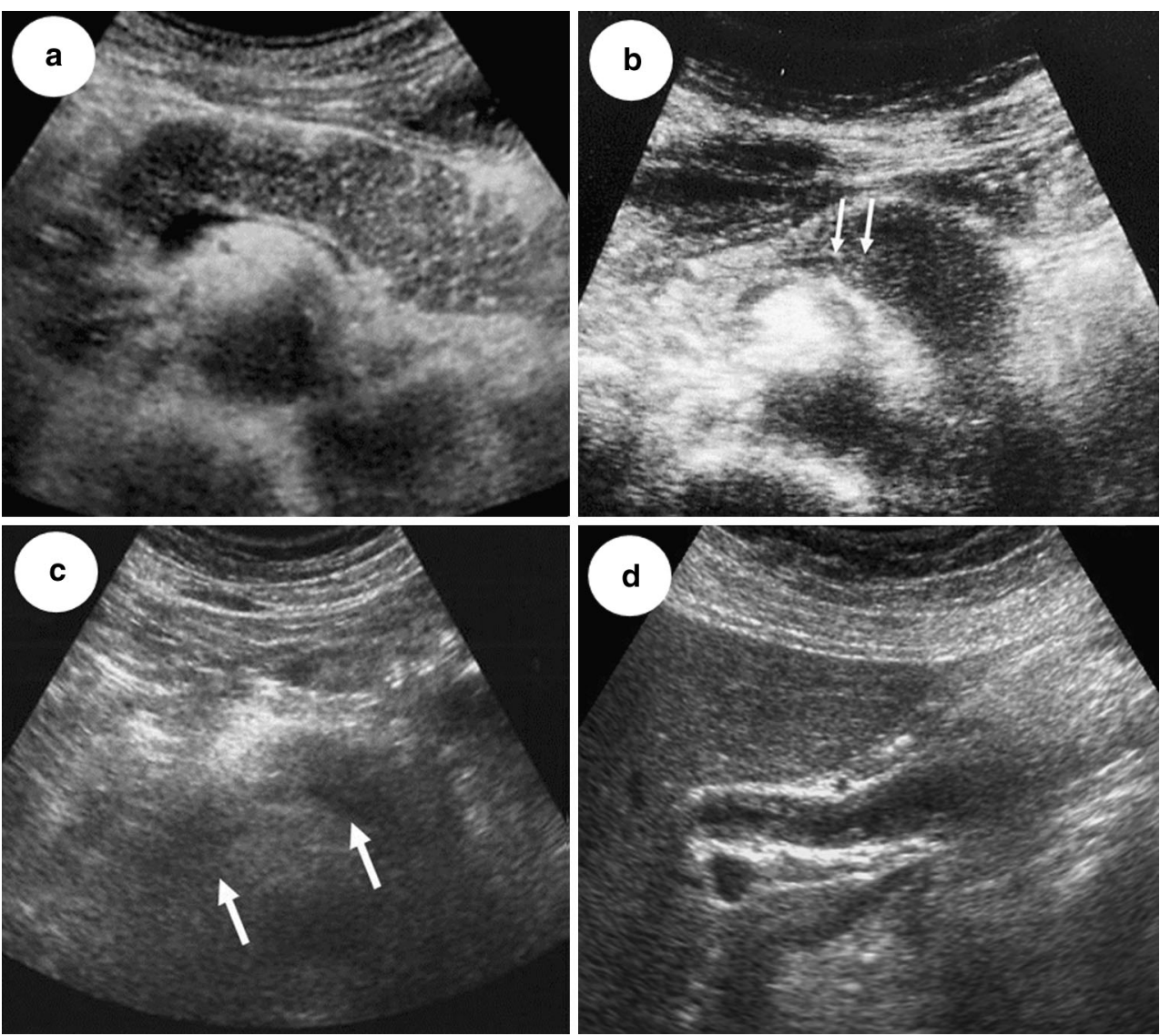

is seen in most cases. The enlarged area shows a low echo image, in some cases with scattered high echo spots [44, 45]. A segmentally/focally enlarged pancreas must be distinguished from pancreatic cancer or mass-forming pancreatitis through a differential diagnosis. Although dilatation of the main pancreatic duct is not seen in most cases, some patients may show minor dilation, which makes the differential diagnosis difficult. Conversely, if the main duct is found to penetrate through the mass (Fig. 1b), duct penetration may be a useful sign to rule out pancreatic cancer [46]. In some cases, there may be many low echo mass images in the pancreatic parenchyma (Fig. 1c), which makes it difficult to differentiate AIP from malignant lymphoma or metastatic pancreatic tumors.

It has been reported that around $60 \%$ of patients with AIP show a thickened bile duct wall [45] (Fig. 1d). A thickened bile duct wall is characterized by layered or parenchymal low echo wall thickening [47, 48]. There have been some cases where the thick wall centered around the extrahepatic bile duct extends over to the intrahepatic bile duct or gallbladder [47]. The wall thickening has been studied in detail with intraductal ultrasonography (IDUS) $[45,48]$. Although wall thickening of narrowed areas is not clearly visible in US, since areas other than the narrowed area show thickening of the internal low-echo layer while maintaining the high-echo image for the outer area, it is assumed that the thickening is happening on the bile duct wall itself [49].

Some recent reports have discussed the usefulness of contrast-enhanced ultrasonography in the diagnosis to differentiate AIP from pancreatic cancer [50-52]. Reports have shown that while in the case of pancreatic cancer, only the rim of the mass was stained with the presence of tumor vessels; in the case of AIP, the entire mass was stained without presence of tumor vessels. However, reports have also shown that for AIP, findings varied depending on the stage of the disease. The areas of stronger inflammation and immature fibrosis were stained strongly, whereas the areas of weaker inflammation and older fibrosis were stained weakly [52].

\section{CQ-I-7. What are the characteristic findings of abdominal computed tomography (CT)?}

- Abdominal CT images of patients with AIP show a diffusely or locally enlarged pancreas. The dynamic CT shows a distinctive delayed enhancement pattern with various images depending on the activity or stage of the disease. (Level of recommendation: A).

- If a capsule-like rim is observed, the patient is highly suspected of having AIP. (Level of recommendation: A). 
Description Typical AIP exhibits a diffusely enlarged pancreas [3]. The pancreatic parenchyma is replaced by fibrosis, which causes a reduced enhancement effect during the "pancreatic parenchymal phase" and shows less absorption compared to a normal pancreas. Due to the delayed enhancement in the area of fibrosis, a certain level of enhancement is seen in the "portal phase" and the enhancement continues into the "delayed phase" where the enhancement becomes stronger than in a normal pancreas. Consequently, the dynamic CT enhancement pattern of AIP shows a slow and delayed enhancement pattern. However, because weak fibrosis shows an enhancement pattern similar to normal pancreas, even in the absence of delayed enhancement, the possibility of AIP cannot be ruled out [44].

A "capsule-like rim" is a relatively distinctive CT feature of AIP (Fig. 2a, b) [44, 53]. It is a band-like structure that appears to surround all or part of the lesions; it shows lower absorption than pancreatic parenchyma of the lesion during the pancreatic parenchymal phase, and a

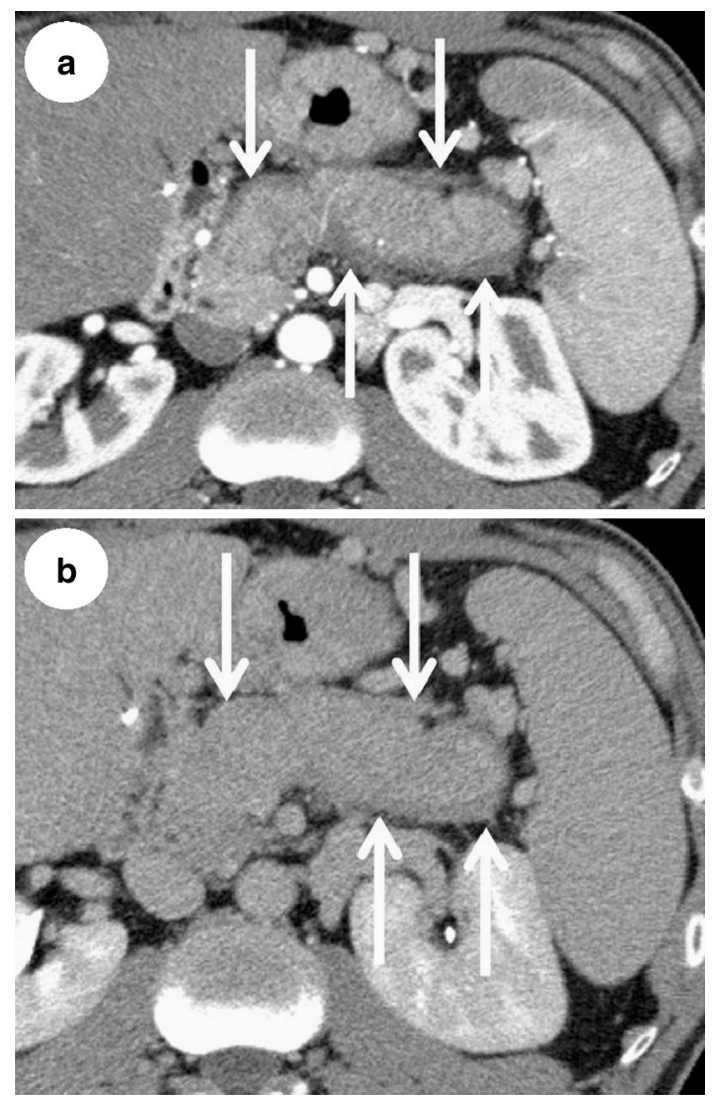

Fig. 2 Abdominal CT in AIP. a Parenchymal phase: The pancreatic parenchyma shows irregularly reduced enhancement. The marginal zone of the pancreas shows capsule-like rim with more reduced enhancement (arrows). b Delayed phase: the enhancement in the parenchyma and capsule-like rim become stronger in the delayed phase.(arrows) delayed enhancement pattern with dynamic CT [44, 53]. While these findings may indicate fibrosis of the rim of the lesion, the frequency of such findings varies depending on the report [53, 54]. This finding, however, is specific to AIP and is not seen in any other diseases. If a capsule-like rim is observed, the chance of the patient having AIP is high. A locally enlarged pancreas is an especially useful sign to distinguish AIP from pancreatic cancer [44, 53]. (Refer to CQ-III-3) Although an actual positive rate of capsule-like rim in AIP on CT images remains unclear because of few reports, a recent single study suggested $48 \%$ [55] .

AIP exhibits many different CT images. Many AIP patients are elderly. Because their pancreases are atrophied to begin with, a pancreas enlarged from AIP is not clearly seen. In some cases, the pancreatic enlargement is verified only after steroid treatment by comparing the size before and after the treatment. There are cases where no abnormality other than a minor diffusely enlarged pancreas is found, partial dilatation of the main duct is pronounced, cystic lesions that appear to be pseudocysts are involved, or the pancreatic parenchyma shows obvious calcification. It must be realized that the absence of typical CT images cannot be the reason to exclude AIP from consideration [44, 53]. A recent study reported that pancreatic volumetric perfusion was attenuated in AIP patients, and improved after the steroid treatment. This suggested that the perfusion CT may be useful in evaluating therapeutic effects [56].

CQ-I-8. Can magnetic resonance cholangiopancreatography (MRCP) evaluate narrowing of the main pancreatic duct in AIP?

- MRI images of AIP show a diffusely enlarged pancreas with distinctive characteristics, such as a low signal on T1-weighted images and a delayed enhancement pattern on dynamic MRI images. (Level of recommendation: A).

- A "capsule-like rim" reflects strong fibrosis of the peripancreatic lesion, which is highly specific for AIP. (Level of recommendation: A).

- At this moment, MRCP is not recommended for the accurate evaluation of the narrowing of the main pancreatic duct. (Level of recommendation: B).

Description Like other image examinations, MR images show a diffusely or locally enlarged pancreas in cases of AIP [3]. The basic MR images used to examine AIP are T1-weighted images, T2-weighted images, and dynamic MRI; AIP lesions show a low signal on T1-weighted images. A normal pancreas shows a higher signal than the liver on T1-weighted images; therefore, a pancreas showing a lower signal than the liver is judged to be abnormal. However, since a low signal is also seen in pancreatic 
cancer or normal chronic pancreatitis, it is not a characteristic finding of AIP $[44,53]$. The T2-weighted images may show a slightly lower signal in strong fibrosis and a slightly stronger signal in weak fibrosis [44, 53]. Meanwhile, the dynamic MR image shows a delayed enhancement pattern, as seen in the dynamic CT [44, 53]. (Refer to CQ-I-7).

Because a capsule-like rim is observed in MRI in about $36 \%$ of patients with AIP [57], its presence can be used as a supplementary diagnostic tool for the disease. The capsule-like rim is extracted as a low signal on T2weighted images reflecting strong fibrosis. Dynamic MR images show a delayed enhancement pattern [53, 54].

Although MRCP is useful in evaluating the narrowing of the main pancreatic duct in cases of multiple stenosis (skipped lesions), unremarkably dilated upper stream, or steroidal improvement, it is currently difficult to use MRCP images for the diagnosis of AIP [3]. However, recent significant progress in MRI technology has made it possible to extract images of the normal main pancreatic duct by 3-D MRCP without fail. Therefore, if the main pancreatic duct is not extracted by 3-D MRCP, it may be an indication of prominent stenosis. Since further image quality improvement can be expected for MRCP with the introduction of 3-Stela MRI technology, it is possible that MRCP will be used to evaluate the therapeutic effect or monitor the progress of AIP in the future [44, 53]. Recently, diffusion-weighted MRI (DWI) has been reported to be useful for detecting AIP by distinguishing it from pancreatic cancer, and for evaluating the effect of steroid therapy [58-60].

\section{CQ-I-9. What are the characteristic findings of positron emission tomography (PET) and gallium-scintigram in} AIP?

- Patients with AIP show accumulation of gallium citrate (Ga-67) and fluorine-18 fluorodeoxyglucose (FDG) in pancreatic and extra-pancreatic lesions, which disappears shortly after steroid treatment. The characteristic accumulation pattern and kinetics following the steroid treatment can be used for the diagnosis of the disease. (Level of recommendation: B).

Description Gallium scintigraphy shows accumulation of Ga-67 in localized pancreatic lesions in patients with AIP. Previously, some such cases were diagnosed as pancreatic malignant lymphoma [61]. The accumulation of Ga-67 is found not only in pancreatic lesions but also in extrapancreatic lesions such as in the hilar lymph nodes, lacrimal gland, or salivary gland. The accumulation is found in about $70 \%$ of pancreatic lesions and hilar lymph nodes, and about $20 \%$ of lacrimal/salivary glands. The
Fig. 3 Pancreatogram in AIP. a Diffuse type: diffusely irregular narrowing of the main pancreatic duct is seen from the pancreas head to tail. b Segmental type: irregular narrowing of the main pancreatic duct is seen from the pancreas body to tail. c Focal type: irregular narrowing of the main pancreatic duct is seen in the pancreas head without dilation of upperstream. d Multiple narrowing type: discontinuous and multiple narrowing of the main pancreatic duct (skip lesions) are seen in the pancreatic head and body
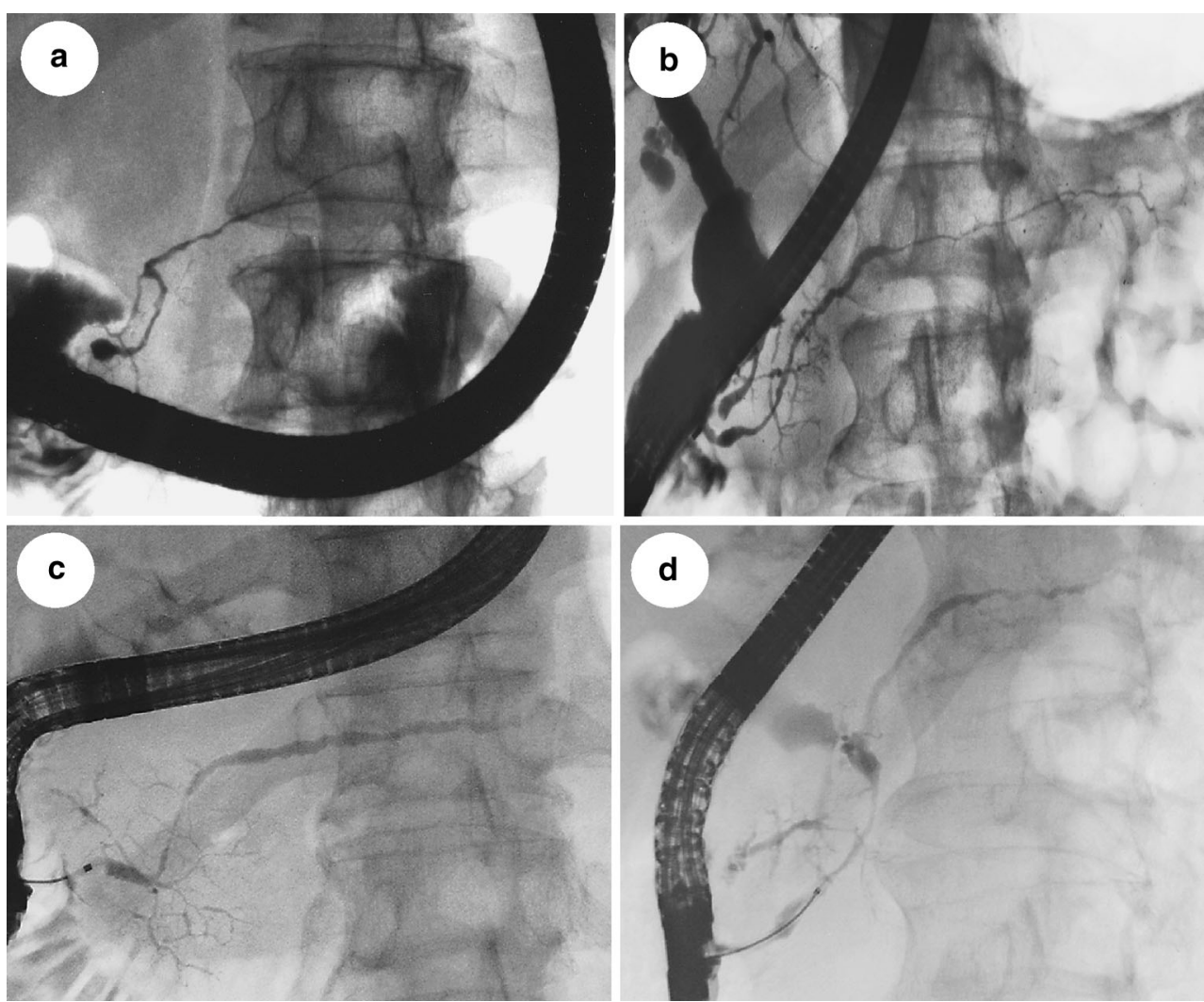
accumulation reflects high disease activity and disappears quickly after steroid treatment [62]. Therefore, the distribution of Ga-67 accumulation and the kinetics after steroid treatment can be used for the diagnosis of AIP.

FDG-PET (fluorine-18 fluorodeoxyglucose positron emission tomography) is useful for the diagnosis of pancreatic cancer. However, high accumulation of FDG ( $90 \%$ or higher) is also observed in patients with AIP. In these cases, the accumulation corresponds to the prominent inflammatory cell infiltration areas [63-66]. FDG also accumulates in extra-pancreatic lesions such as in the salivary gland, a wide range of lymph node lesions, retroperitoneal fibrosis, and the prostate gland [67-69]. Accumulated FDG in pancreatic or extra-pancreatic areas disappears quickly after steroid treatment [66]. The following two criteria are useful in distinguishing AIP from pancreatic cancer: extensive or multiple accumulations of FDG in the pancreas, or distinctive accumulation in extra-pancreatic lesions in the salivary gland, retroperitoneal fibrosis, or prostate gland [66, 67]. Although the disappearance of FDG following steroid treatment is reported to be used as a criterion to distinguish AIP from pancreatic cancer [70], a facile steroid trial should be carefully performed after a negative work-up of malignancy, because a follow-up study of FDG-PET is not supported by Japanese medical insurance.

\section{CQ-I-10. What are the characteristic findings of endo- scopic retrograde cholangiopancreatography (ERCP) in} AIP?

- ERCP shows narrowing of the main pancreatic duct characteristic of AIP. (Level of recommendation: A).

- AIP may be associated with stenosis of the bile duct. (Level of recommendation: A).
Fig. 4 Histopathological and Immunohistochemical findings in AIP (LPSP:

Lymphoplasmacytic sclerosing pancreatitis): a fibrosis, prominent infiltration of lymphocytes and plasmacytes (LPSP) are seen. b. Numerous IgG4-positive cells in LPSP are seen. c Storiform fibrosis. An irregularly whorled pattern of fibrosis (storiform fibrosis) with various degrees of infiltration of inflammatory cells, small fusiform cells and fibrotic changes is observed.

d Obliterative phlebitis. Stenosis or obstruction of vessels with infiltration of lymphocytes and plasmacytes, and fibrosis is seen.

e Circumferential inflammation of LPSP around duct epithelium with stenotic lumen
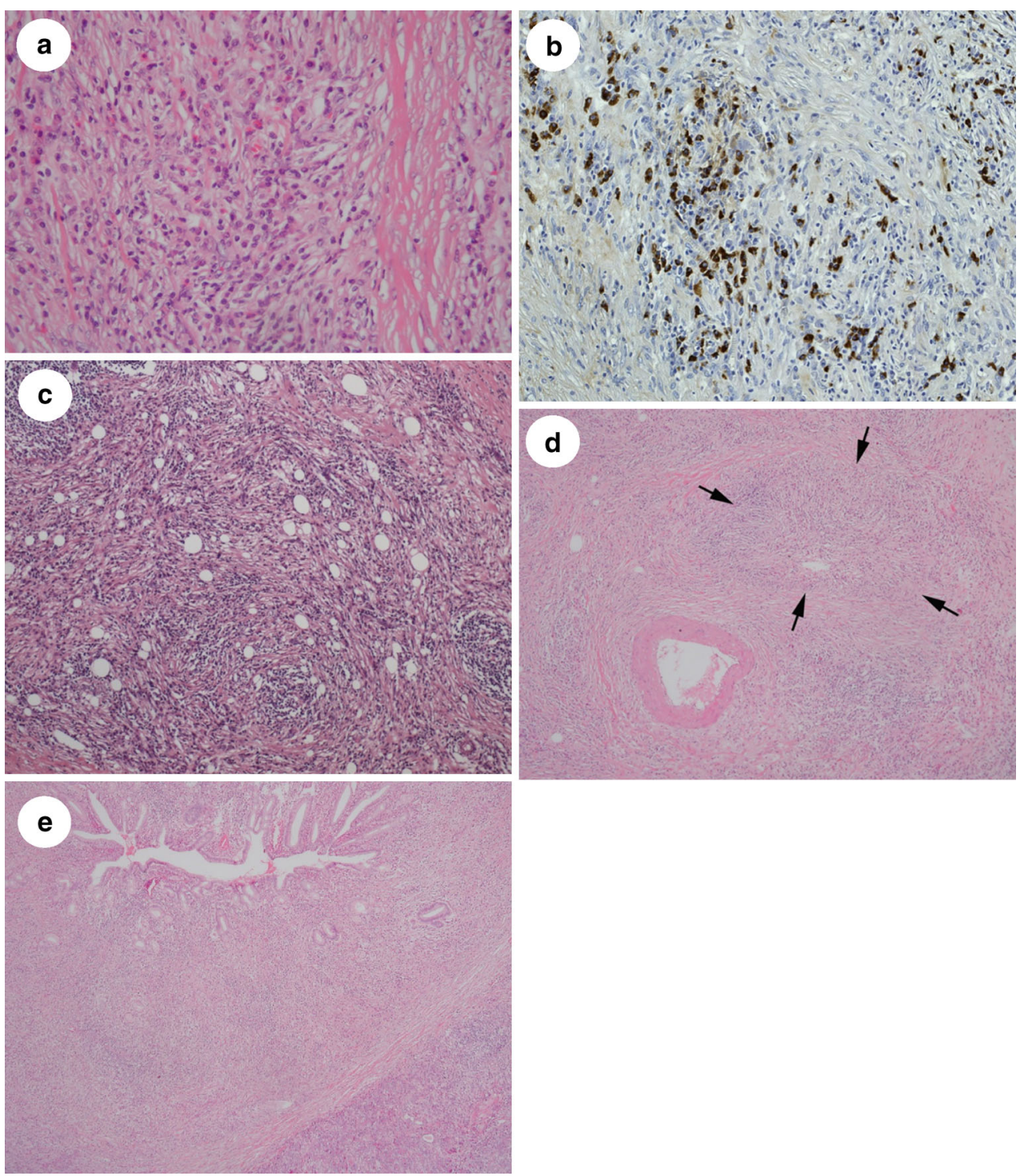
Table 3 Clinical diagnostic criteria for autoimmune pancreatitis 2011

A. Diagnostic criterion

I. Enlargement of the pancreas:

a. Diffuse enlargement

b. Segmental/focal enlargement

II. ERP (endoscopic retrograde pancreatography) shows irregular narrowing of the main pancreatic duct

III. Serological findings

Elevated levels of serum IgG4 ( $\geq 135 \mathrm{mg} / \mathrm{dl}$ )

IV. Pathological findings: among i)-iv) listed below,

a. Three or more are observed

b. Two are observed

i) Prominent infiltration and fibrosis of lymphocytes and plasmacytes

ii) Ten or more diffuse IgG4-positive plasmacytes per high-power microscope field

iii) Storiform fibrosis

iv) Obliterative phlebitis

V. Other organ involvement (OOI): sclerosing cholangitis, sclerosing dacryoadenitis/ sialoadenitis, retroperitoneal fibrosis

a. Clinical lesions

Extra-pancreatic sclerosing cholangitis, sclerosing dacryoadenitis/sialoadenitis (Mikulicz disease), or retroperitoneal fibrosis can be diagnosed with clinical and image findings

b. Pathological lesions

Pathological examination shows characteristic features of sclerosing cholangitis, sclerosing dacryoadenitis/sialoadenitis, or retroperitoneal fibrosis

$<$ Option $>$ Effectiveness of steroid therapy

A specialized facility may include in its diagnosis the effectiveness of steroid therapy, once pancreatic or bile duct cancers have been ruled out. When it is difficult to differentiate from malignant conditions, it is desirable to perform cytological examination using an endoscopic ultrasound-guided fine needle aspiration (EUS-FNA). Facile therapeutic diagnosis by steroids should be avoided unless the possibility of malignant tumor has been ruled out by pathological diagnosis

B. Diagnosis

I. Definite diagnosis

(1) Diffuse type

(2) Segmental/focal type

$\mathrm{I} a+<\mathrm{III} / \mathrm{IVb} / \mathrm{V}(\mathrm{a} / \mathrm{b})>$

I $b+$ II + two or more of $<$ III/IV b/V (a/b) $>$

I $\mathrm{b}+\mathrm{II}+<\mathrm{III} / \mathrm{IV} \mathrm{b} / \mathrm{V}(\mathrm{a} / \mathrm{b})>+$ Option

(3) Definite diagnosis by histopathological study

IV a

II. Probable diagnosis

Segmental/focal type:

$\mathrm{I} b+\mathrm{II}+<\mathrm{III} / \mathrm{IV}$ b/V $(\mathrm{a} / \mathrm{b})>$

III. Possible diagnosis*

Diffuse type:

$\mathrm{I} \mathrm{a}+\mathrm{II}+$ Option

Segmental/focal type:

I b + II + Option

When a patient with a focal/segmental image of AIP on CT/MRI without ERCP findings fulfill more than one of III, IVb and V(a/b) criteria, he/ she can be diagnosed as possible AIP only after the negative workup for malignancy by EUS-FNA, and confirmed as probable one by an optional steroid response

Possible diagnosis*: A case may be possibly type 2, although it is extremely rare in Japan

"+" refers to "and", and "/" refers to "or" 
Table 4 Diagnosis of definitive and probable type 1 AIP using ICDC

\begin{tabular}{llll}
\hline Diagnosis & Primary basis for diagnosis & Imaging evidence & Collateral evidence \\
\hline Definitive type 1 AIP & Histology & Typical/indeterminate & Histologically confirmed LPSP (level 1 H) \\
& Imaging & Typical & Any non-D level 1/level 2 \\
& & Indeterminate & Two or more from level 1 (+level 2 D*) \\
& \multirow{2}{*}{ Response to steroid } & Indeterminate & Level 1 S/OOI + Rt or level 1 D + level 2 S/OOI/H + Rt \\
Probable type 1 AIP & & Indeterminate & Level 2 S/OOI/H $+\mathrm{Rt}$ \\
\hline
\end{tabular}

*Level $2 \mathrm{D}$ is counted as level 1 in this setting

Table 5 Level 1 and Level 2 criteria for type 1 AIP

\begin{tabular}{|c|c|c|c|}
\hline & Criterion & Level 1 & Level 2 \\
\hline $\mathrm{P}$ & $\begin{array}{l}\text { Parenchymal } \\
\text { imaging }\end{array}$ & $\begin{array}{l}\text { Typical: } \\
\text { Diffuse enlargement with delayed enhancement } \\
\text { (sometimes associated with rim-like enhancement) }\end{array}$ & $\begin{array}{l}\text { Indeterminate (including atypical }{ }^{\dagger} \text { ): } \\
\text { Segmental/focal enlargement with delayed } \\
\text { enhancement }\end{array}$ \\
\hline D & $\begin{array}{l}\text { Ductal imaging } \\
\text { (ERP) }\end{array}$ & $\begin{array}{l}\text { Long ( }>1 / 3 \text { length of the main pancreatic duct) or multiple strictures } \\
\text { without marked upstream dilatation }\end{array}$ & $\begin{array}{l}\text { Segmental/focal narrowing without marked } \\
\text { upstream dilatation (duct size, }<5 \mathrm{~mm} \text { ) }\end{array}$ \\
\hline $\mathrm{S}$ & Serology & IgG4, $>2 \times$ upper limit of normal value & $\operatorname{IgG} 4,1-2 \times$ upper limit of normal value \\
\hline OOI & $\begin{array}{l}\text { Other organ } \\
\text { involvement }\end{array}$ & $\begin{array}{l}\text { a or b } \\
\text { a. Histology of extrapancreatic organs } \\
\text { Any three of the following: } \\
\text { (1) Marked lymphoplasmacytic infiltration with fibrosis and without } \\
\text { granulocytic infiltration } \\
\text { (2) Storiform fibrosis } \\
\text { (3) Obliterative phlebitis } \\
\text { (4) Abundant (>10 cells/HPF) IgG4-positive cells } \\
\text { b. Typical radiological evidence } \\
\text { At least one of the following: } \\
\text { (1) Segmental/multiple proximal (hilar/intrahepatic) } \\
\text { or proximal and distal bile duct stricture } \\
\text { (2) Retroperitoneal fibrosis }\end{array}$ & $\begin{array}{l}\text { a or b } \\
\text { a. Histology of extrapancreatic organs including } \\
\text { endoscopic biopsies of bile duct }{ }^{\ddagger} \text { : } \\
\text { Both of the following: } \\
\text { (1) Marked lymphoplasmacytic infiltration } \\
\text { without granulocytic infiltration } \\
\text { (2) Abundant (>10 cells/HPF) IgG4-positive } \\
\text { cells } \\
\text { b. Physical or radiological evidence } \\
\text { At least one of the following: } \\
\text { (1) Symmetrically enlarged salivary/lachrymal } \\
\text { glands } \\
\text { (2) Radiological evidence of renal involvement } \\
\text { described in association with AIP }\end{array}$ \\
\hline $\mathrm{H}$ & $\begin{array}{l}\text { Histology of the } \\
\text { pancreas }\end{array}$ & $\begin{array}{l}\text { LPSP (core biopsy/resection) } \\
\text { At least } 3 \text { of the following: } \\
\text { (1) Periductal lymphoplasmacytic infiltrate without granulocytic } \\
\text { infiltration } \\
\text { (2) Obliterative phlebitis } \\
\text { (3) Storiform fibrosis } \\
\text { (4) Abundant ( }>10 \text { cells/HPF) IgG4-positive cells }\end{array}$ & $\begin{array}{l}\text { LPSP (core biopsy) } \\
\text { Any } 2 \text { of the following: } \\
\text { (1) Periductal lymphoplasmacytic infiltrate } \\
\text { without granulocytic infiltration } \\
\text { (2) Obliterative phlebitis } \\
\text { (3) Storiform fibrosis } \\
\text { (4) Abundant ( }>10 \text { cells/HPF) IgG4-positive } \\
\text { cells }\end{array}$ \\
\hline \multicolumn{2}{|c|}{$\begin{array}{l}\text { Response to steroid } \\
\text { (Rt)* }\end{array}$} & \multicolumn{2}{|c|}{$\begin{array}{l}\text { Diagnostic steroid trial } \\
\text { lly demonstrable resolution or marked improvement in pancreatic/extrapancreatic }\end{array}$} \\
\hline
\end{tabular}

*Diagnostic steroid trial should be conducted carefully by pancreatologists with caveats (see text) only after negative workup for cancer including endoscopic ultrasound-guided fine needle aspiration

$\dagger$ Atypical: Some AIP cases may show low-density mass, pancreatic ductal dilatation, or distal atrophy. Such atypical imaging findings in patients with obstructive jaundice and/or pancreatic mass are highly suggestive of pancreatic cancer. Such patients should be managed as pancreatic cancer unless there is strong collateral evidence for AIP, and a thorough workup for cancer is negative (see algorithm)

* Endoscopic biopsy of duodenal papilla is a useful adjunctive method because ampulla often is involved pathologically in AIP

Description ERCP shows narrowing of the main pancreatic duct, which is characteristic of AIP. This finding is used as the basis for diagnosis [1]. Narrowing of the pancreatic duct is usually diagnosed from ERCP images. The narrowing of the pancreatic duct is "unlike the obstruction or stenosis, as the narrowing extends to certain 
degree and the duct diameter is smaller (narrower) than normal, with some irregularities" $[1,71]$ (Fig. 3a).

The Clinical Diagnostic Criteria of Autoimmune Pancreatitis 2011 (JPS-2011) states that diagnosis of the disease requires pancreatic images showing "the distinctive narrowing of the main pancreatic duct", where the narrowing may be diffuse or local. The range of narrowing varies. The typical case exhibits narrowing over one third of the entire pancreatic duct (Fig. 3b). Even when the narrowing is localized to less than one third of the entire duct, in most cases no significant dilatation is observed above the narrowed area upstream of the main duct [72-75] (Fig. 3c).

There are, however, other cases where the narrowing is localized to less than one third, or the lesions are multiplestenotic (skip lesions) (Fig. 3d) [73, 74]. If the narrowing is localized, it is necessary to consider differentiating the disease from pancreatic cancer $[4,75,76]$. Typical pancreatic duct features of AIP visible in ERCP images, such as side branch arising from narrowed portion or multiple stenosis of the main pancreatic duct, are useful for differential diagnosis from pancreatic cancer. Short narrowing images of the main pancreatic duct less than $3 \mathrm{~cm}$ are impossible $[4,75,76]$.

About $80 \%$ of patients with AIP show stenosis of the bile duct [77-81]. Although most of the stenosis is found in the lower bile duct, it can also be detected in the extrahepatic or intra-hepatic bile ducts [77-81].

\section{CQ-I-11. What are the characteristic histopathological findings in AIP?}

- As histopathological findings of type 1 AIP are characteristic, typical ones can be diagnosed by themselves without clinical informations (Level of recommendation: A).

- Histopathological findings of AIP are characterized by fibrosis with strong lymphoplasmacytic infiltration, abundant infiltration of IgG4-positive plasmacytes, storiform fibrosis, obliterative phlebitis, and periductal inflammation of inflammatory cells. (Level of recommendation: A).

Table 6 Diagnosis of Definitive and Probable Type 2 AIP, and AIP-not otherwise specified using ICDC

\begin{tabular}{lll}
\hline Diagnosis & Imaging evidence & Collateral evidence \\
\hline Definitive type 2 AIP & $\begin{array}{c}\text { Typical/ } \\
\text { indeterminate }\end{array}$ & $\begin{array}{c}\text { Histologically confirmed IDCP (level 1 H) or clinical inflammatory bowel disease + level 2 } \\
\mathrm{H}+\mathrm{Rt}\end{array}$ \\
$\begin{array}{c}\text { Typical/ } \\
\text { indeterminate }\end{array}$ & Level 2 H/clinical inflammatory bowel disease + Rt \\
$\begin{array}{c}\text { AIP-not otherwise } \\
\text { specified }\end{array}$ & $\begin{array}{c}\text { Typical/ } \\
\text { indeterminate }\end{array}$ & D 1/2 + Rt \\
\hline
\end{tabular}

Table 7 Level 1 and Level 2 Criteria for Type 2 AIP

\begin{tabular}{|c|c|c|c|}
\hline & Criterion & Level 1 & Level 2 \\
\hline \multirow[t]{2}{*}{$\mathrm{P}$} & \multirow{2}{*}{$\begin{array}{l}\text { Parenchymal } \\
\text { imaging }\end{array}$} & Typical: & Indeterminate (including atypical ${ }^{\dagger}$ ): \\
\hline & & $\begin{array}{l}\text { Diffuse enlargement with delayed enhancement } \\
\text { (sometimes associated with rim-like enhancement) }\end{array}$ & $\begin{array}{l}\text { Segmental/focal enlargement with delayed } \\
\text { enhancement }\end{array}$ \\
\hline $\mathrm{D}$ & $\begin{array}{l}\text { Ductal imaging } \\
\text { (ERP) }\end{array}$ & $\begin{array}{l}\text { Long ( }>1 / 3 \text { length of the main pancreatic duct) or multiple strictures } \\
\text { without marked upstream dilatation }\end{array}$ & $\begin{array}{l}\text { Segmental/focal narrowing without marked } \\
\text { upstream dilatation (duct size, }<5 \mathrm{~mm} \text { ) }\end{array}$ \\
\hline OOI & $\begin{array}{l}\text { Other organ } \\
\text { involvement }\end{array}$ & & $\begin{array}{l}\text { Clinically diagnosed inflammatory bowel } \\
\text { disease }\end{array}$ \\
\hline \multirow[t]{4}{*}{$\mathrm{H}$} & \multirow{4}{*}{$\begin{array}{l}\text { Histology of the } \\
\text { pancreas } \\
\text { (core biopsy/ } \\
\text { resection) }\end{array}$} & IDCP: & Both of the following: \\
\hline & & Both of the following: & \multirow{3}{*}{$\begin{array}{l}\text { (1) Granulocytic and lymphoplasmacytic acinar } \\
\text { infiltrate } \\
\text { (2) Absent or scant }(0-10 \text { cells/HPF) IgG4- } \\
\text { positive cells }\end{array}$} \\
\hline & & (1) Granulocytic infiltration of duct wall (GEL) with or without & \\
\hline & & (2) Absent or scant ( $0-10$ cells/HPF) IgG4-positive cells & \\
\hline \multirow{2}{*}{\multicolumn{2}{|c|}{$\begin{array}{l}\text { Response to steroid } \\
\text { (Rt)* }\end{array}$}} & \multicolumn{2}{|l|}{ Diagnostic steroid trial } \\
\hline & & \multicolumn{2}{|c|}{ Rapid ( $\leq 2$ weeks) radiologically demonstrable resolution or marked improvement in manifestations } \\
\hline
\end{tabular}

*Diagnostic steroid trial should be conducted carefully by pancreatologists with caveats (see text) only after negative workup for cancer including endoscopic ultrasound-guided fine needle aspiration

${ }^{\dagger}$ Atypical: Some AIP cases may show low-density mass, pancreatic ductal dilatation, or distal atrophy. Such atypical imaging findings in patients with obstructive jaundice and/or pancreatic mass are highly suggestive of pancreatic cancer. Such patients should be managed as pancreatic cancer unless there is strong collateral evidence for AIP, and a thorough workup for cancer is negative (see algorithm) 
- Histopathological findings of type 2 AIP are characterized by infiltration of granulocytes into the epithelium and lumen of the interlobular pancreatic duct. Infiltration of IgG4-positive plasmacytes is rarely seen (Level of recommendation: A).

Description As histopathological findings of type 1 AIP called "lymphoplasmacytic sclerosing pancreatitis (LPSP) [21]" are characteristic, typical ones can be diagnosed by themselves without clinical information. The major histological findings are (i) prominent infiltration of lymphoplasmacytes without granulocytic infiltration and fibrosis (Fig. 4a); (ii) abundant ( $>10$ cells/HPF) IgG4-positive cells (Fig. 4b); (iii) storiform fibrosis (Fig. 4c); (iv) obliterative phlebitis (Fig. 4d); and (v) periductal infiltration of inflammatory cells (Fig. 4e); and the fibrosis associated with prominent infiltration of lymphocytes and plasmacytes [6-11, 82-86]. When three of the first four (i-iv) histologic items fulfil the Japanese clinical diagnostic criteria (JPS-2011) [10, 11]' or three of the last four (ii-v) fulfil the ICDC [9], definitive diagnosis can be made based on histology alone.

Type 1 AIP is considered to be a pancreatic manifestation of IgG4-RD [17-20, 87-89]. Abundant infiltration of IgG4positive plasmacytes is characteristic, but not specific for
IgG4-RD. Because a few cases have been reported in which IgG4-positive plasmacytes appear in patients with pancreatic cancer or alcoholic pancreatitis, IgG4-positive plasmacytes cannot be used as the sole basis for the diagnosis of AIP [1720, 87-89]. Therefore, histopathological findings as well as infiltration of IgG4-positive plasmacytes are important in the diagnosis of IgG4-RD [17-20, 87-89].

The histopathological diagnosis of type 1 AIP is easy with a resected specimen, and difficult with a biopsy specimen. Although many investigators reported that diagnostic values by cytological or histological diagnosis using EUS-FNA are low, those by histological diagnosis using EUS-trucut biopsy are high [90-92]. However, a recent study suggested that histological diagnosis by EUSFNA using $22 \mathrm{G}$ needles was useful [93].

Type 2 AIP is idiopathic pancreatitis. It is characterized by the infiltration of neutrophils into the epithelium and/or lumen of interlobular pancreatic ducts, and is referred to as either ICDP or AIP with GEL [22, 23, 82, 85]. To make a definitive diagnosis of type 2 AIP, histopathological criteria are the gold standard due to the lack of specific images and serum markers [9]. In some cases, however, a few IgG4-positive plasmacytes (more than 10 cells/HPF in rare cases) may be observed.

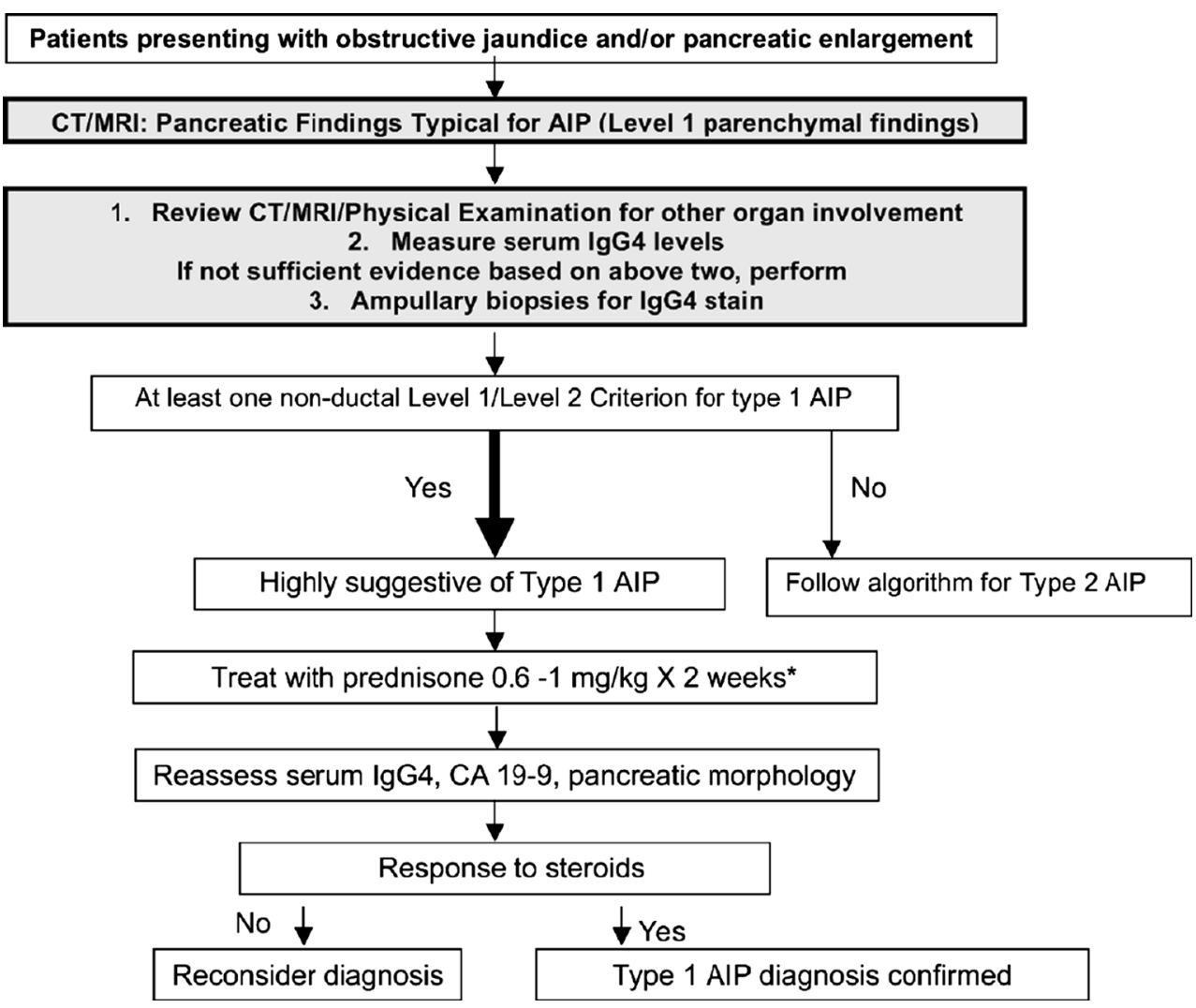

Fig. 5 Algorithm to diagnose type 1 AIP in subjects presenting with obstructive jaundice and/or pancreatic enlargement. This schematic drawing shows a flow to diagnose type 1 AIP with typical diffuse enlargement of the pancreas on CT/MRI (level 1 parenchymal findings) 


\section{CQ-I-12. How should AIP be diagnosed?}

- A comprehensive diagnosis must be performed based on pancreatic image findings, serological findings, histopathological findings, other organ involvement, and steroid treatment effects. (Level of recommendation: A).

- The international consensus diagnostic criteria (ICDC) for AIP can differentiates between type 1 and type 2 . (Level of recommendation: A).

- In Japan, most AIP cases are type 1, as defined by the Japanese Clinical Diagnostic Criteria 2011 (JPS-2011). (Level of recommendation: A).

Description The Japan Pancreas Society took the initiative to propose the world's first clinical diagnostic criteria for AIP in 2002 [3]. The criteria were revised in 2006 by the joint efforts of the Japan Pancreas Society and the Research Committee for Intractable Pancreas Disease supported by the Ministry of Health and Welfare of Japan (RCIPD-MLHWJ) [4, 5]. The Japanese diagnostic criteria were designed to be as simple as possible, to be easy for general physicians as well as pancreatologists to use. After a decade of international discussion about diagnostic criteria [94-96], the ICDC enabled the diagnosis of two distinctive subtypes of AIP, type 1 and type2 AIP [9]. However, the ICDC is somewhat complicated for general use. Different from western countries, extremely few cases of type 2 AIP have been confirmed in Japan [6-8, 10, 11]. In response to the proposed ICDC, the JPS and RCIPDMLHWJ revised the clinical diagnostic criteria for AIP in $2011[10,11]$ (Table 3, “Appendix 1").

In contrast to the JPS2002 and JPS2006 criteria, JPS2011 contains the following major diagnostic criteria [10, 11], (1) appearance of diffuse and segmental/focal type in pancreatic parenchymal CT/MRI images or ERCP duct images, (2) a single category without level 1 and 2 classifications in the ICDC (Table 4, 5, 6, 7), (3) IgG4 alone as a serum marker, (4) histopathological criteria for LPSP, (5) sclerosing cholangitis, sclerosing sialoadenitis and retroperitoneal fibrosis as typical OOIs, and (6)

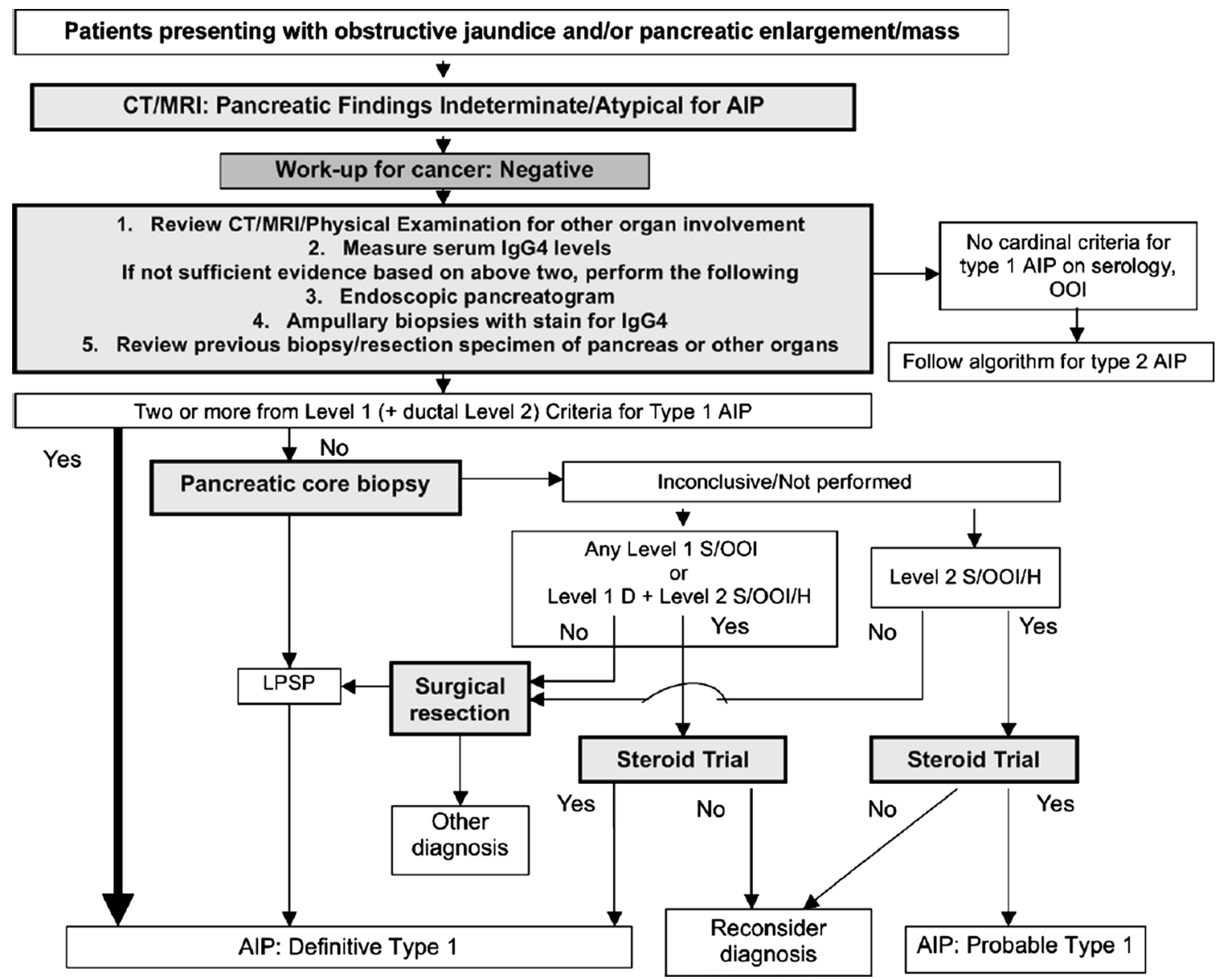

Fig. 6 Algorithm to diagnose type 1 AIP in subjects presenting with obstructive jaundice and/or pancreatic mass. This schematic drawing shows a flow to diagnose type 1 AIP with indeterminate or atypical findings of the pancreas on CT/MRI (level 2 parenchymal findings) 


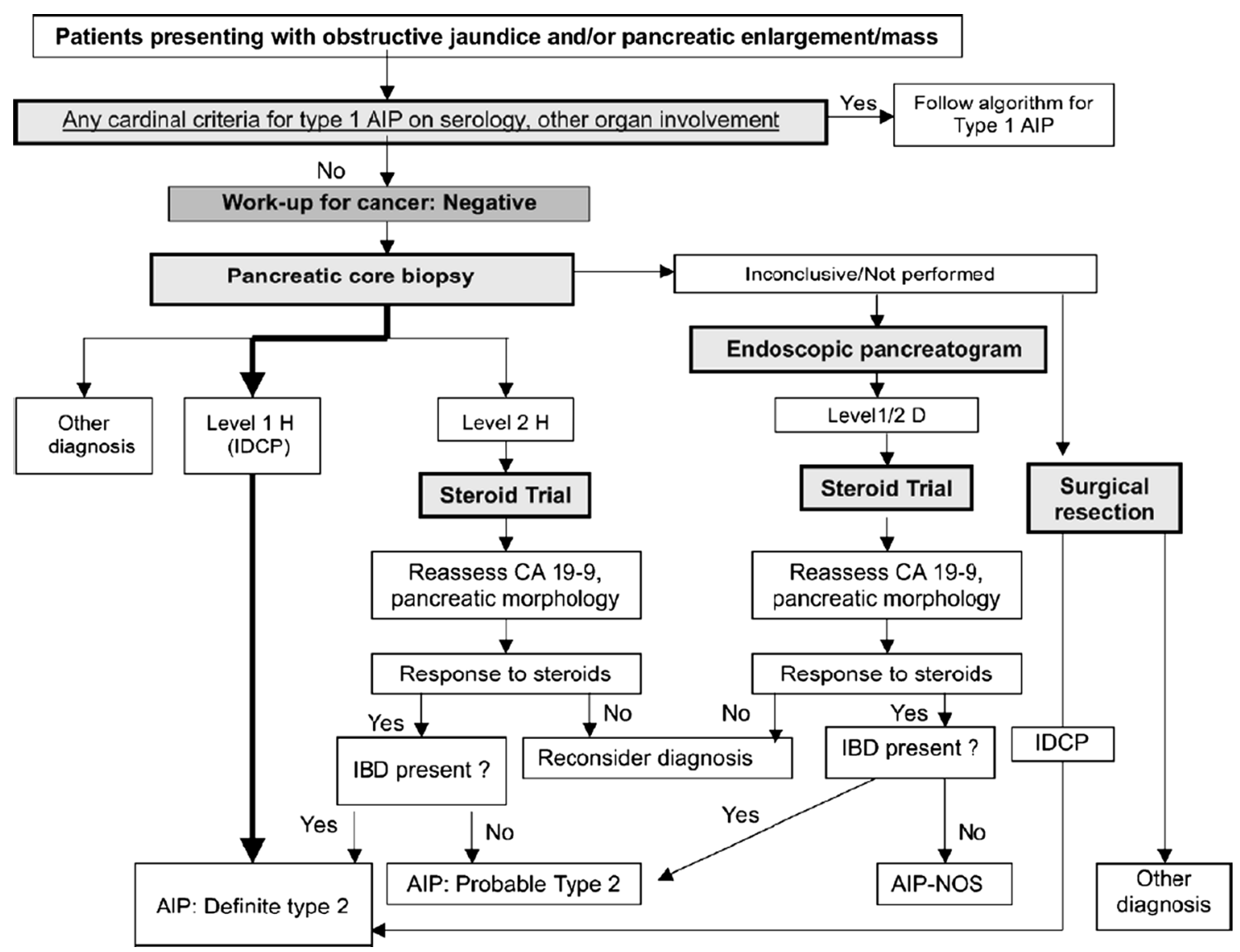

Fig. 7 Algorithm to diagnose type 2 AIP in subjects presenting with obstructive jaundice and/or pancreatic mass. This schematic drawing shows a flow to diagnose type 2 AIP with typical/indeterminate (atypical) findings of the pancreas on CT/MRI (level 1 and 2 parenchymal findings)

response to an optional steroid trial after using EUS-FNA to rule out malignancy. As in JPS-2006, the pancreatic images specific to AIP can be confirmed retrospectively after the diagnosis. Although some patients with pancreatic or biliary malignancy may show high serum levels of IgG4, measurement of serum IgG4 is very useful in the diagnosis of AIP because of higher sensitivity and values compared with other serum markers and malignancies, respectively. There are several reports showing that, if infiltration of IgG4-positive plasmacytes is observed in the biopsy of the duodenal papillary mucosa, the chance of the patient having AIP is high [97, 98]. The inflammation of the duodenal papilla is mainly extended from the pancreas and is excluded from OOIs. Diagnostic algorithms for type 1 and type 2 proposed by the ICDC (Figs. 5, 6, 7) permitted modifications depending on the local expertise.

\section{CQ-I-13. Is facile steroid therapy useful for} a differential diagnosis against pancreatic cancer?

- Response to steroid treatment indicates possible AIP. However, response to steroid treatment does not exclude the possibility of the patient having pancreatic cancer. (Level of recommendation: B).
- In the cases of segmental/focal swelling or tumorforming pancreas, facile steroid treatment should be performed only after a negative work-up for malignancy using ERCP or EUS-FNA cytology. (Level of recommendation: $\mathrm{B}$ ).

In contrast the Mayo's [94] and Korean [95] criteria, the effect of steroid treatment on the pancreas and OOIs are excluded from the Japanese diagnostic criteria. This decision is based on the following reasons: (1) autoimmune hepatitis is the only autoimmune disease that uses the effect of steroid treatment as a diagnostic criteria; (2) the clinical significance is different for the case of autoimmune hepatitis requiring differentiation from chronic hepatitis of other pathogenesis and the case of AIP requiring differentiation from pancreatic or bile duct cancer; (3) no evidence exists to show that the use of steroids does not affect the success of an operation or the long-term prognosis; (4) there is a danger that therapeutic diagnosis by steroid administration may be used as an easy solution to differentiate AIP from malignant tumors such as pancreatic cancer; (5) the standards were established for not only pancreas specialists but also general gastrointestinal internists and general 
practitioners; (6) in Japan, the objective of the diagnostic criteria is not so much to find AIP but rather to eliminate the misdiagnosis of diseases with malignant tumors as often as possible; and (7) there have been reports of AIP associated with pancreatic cancer [3-5]. The ICDC [9] include response to steroid treatment following the Korean [95], and Asian criteria [96] in the criteria proposed jointly by Japan and South Korea in 2008. The JPS-2011 [10, 11] states that if the possibility of pancreatic cancer is excluded by a reliable exclusivediagnosis using EUS-FNA or a similar test, the effect of steroid treatment may be used as diagnostic criteria. Meanwhile, there have been reports of pancreatic cancers associated with AIP [3]. (Refer to Treatment, Prognosis CQ-IV-10, 11.) If a patient responds to steroid treatment, it may suggest that he/she has AIP; however, since it does not exclude malignant tumors, such as pancreatic cancer or deny the association of pancreatic cancer, a simplistic diagnostic treatment must be avoided.

Acknowledgments This study was supported by grant-in-aid for the Intractable Pancreatic Disease from the Ministry of Health, Labour and Welfare of Japan. The authors are grateful to the copyright holders of the artwork in this paper (primarily the Japan Pancreas Society), all of whom kindly provided their consent for reproduction.

Conflict of interest The authors declare that they have no conflict of interest.

\section{Appendix 1: Explanations for the Japanese Clinical Diagnostic Criteria 2011 in Table 3}

\section{Enlarged pancreas}

A diffusely enlarged pancreas with "sausage-like" appearance is highly specific to AIP. However, the problem is how to differentiate a segmentally/focally enlarged pancreas from pancreas cancer. For the definition of enlarged pancreas, many facilities use the criteria suggested by Haaga and consider the pancreas to be enlarged when "the width of the pancreatic head is more than one full transverse diameter of the vertebral body, and the width of the pancreatic tail is more than two-thirds of the transverse diameter of the vertebral body (which are approximately 3 and $2 \mathrm{~cm}$ for the pancreatic head and tail, respectively)." Precise definition is difficult due to agerelated influences; it may be considered as an enlarged pancreas if steroid therapy reduces the pancreas size.

1. Abdominal ultrasound: an enlarged pancreas often shows a hypo-echoic area with scattered hyper-echoic spots in it.
2. Abdominal CT: dynamic CT shows delayed enhancement pattern and a capsule-like rim which are characteristic of AIP.

3. Abdominal MRI: abdominal MRI shows a low signal on a T1-weighed image, and dynamic MRI shows delayed enhancement and a capsule-like rim, which are characteristic of AIP.

4. FDG-PET: abnormal intense uptake is often seen in active lesions; the uptake is reduced after steroid treatment.

II. Narrowing of the main pancreatic duct: Diffuse or segmental/focal irregular narrowing is seen in the main pancreatic duct.

1. Narrowing is referred to as being unlike the obstruction or stenosis, it extends to a certain degree and the duct diameter is smaller than normal, with some irregularities. In a typical case, the narrowing extends over one third $(5 \mathrm{~cm})$ of the entire pancreatic duct; even when the lesion is segmental, no significant dilation is observed above the narrowed area upstream of the main duct. If the narrowing is short (less than about $3 \mathrm{~cm}$ ), it is difficult to differentiate from pancreatic cancer. The presence of side branches arising from narrowed portions of the main pancreatic duct or multiple skip lesions in the main pancreatic duct are effective in differentiating from pancreatic cancer.

2. Examination of pancreatic duct images basically requires a direct pancreatography such as ERP. Currently, magnetic resonance cholangiopancreatography (MRCP) cannot be used for accurate evaluation of the narrowing of the main pancreatic duct; however, it may be used as a reference diagnosis if the main pancreatic duct shows skip lesions.

3. The pancreatic image findings described above may be observed retrospectively from the time of diagnosis.

III. Hematological examination

1. Patients with AIP often show elevated levels of serum gammaglobulin, IgG, or IgG4 and autoantibodies; an elevated level of serum $\operatorname{IgG}(1800 \mathrm{mg} / \mathrm{dl}$ or higher $)$ or IgG4 $(135 \mathrm{mg} / \mathrm{dl}$ or higher) is one criterion for the diagnosis. Although the diagnostic criteria defined in this paper reference only IgG4, since elevated levels of IgG4 are also observed in other diseases, including IgG4-related diseases of other organs (e.g., atopic dermatitis, pemphigus, asthma), it is not necessarily specific to AIP. Serum IgG4 is the best serum marker for differentiating from pancreatic cancer in terms of both sensitivity and specificity. However, caution is advised since elevated levels are also observed in some pancreatic or bile-duct cancers, and there are cases of 
pancreatic cancers associated with AIP. The significance of elevated serum IgG4 in the pathogenesis and pathophysiology of AIP is still not clear.

2. Autoantibodies such as antinuclear antibodies or rheumatoid factor become positive in some cases, from which AIP presence may be suspected.

IV. Pathological findings of the pancreas

AIP shows a specific pathological image, called LPSP, whose typical features are as follows:

1. Prominent infiltration of lymphocytes and plasmacytes, and fibrosis are observed. These are often accompanied by eosinophil infiltration, but without neutrophils infiltration in many cases. Lymphoid follicle formation may also be present. Inflammation is prominent in inter-lobules, intra-lobules, peripancreatic fatty tissues, and around the epithelial cells of the pancreatic duct, however, infiltration of inflammatory cells into the epithelium of the pancreatic duct is rare.

2. Prominent infiltration of IgG4-positive plasmacytes is characteristic of this disease; resected pancreatic specimens show 50 or more positive plasmacytes per high-power microscope field (x400) in most cases. In order to make diagnosis possible for small needle biopsy specimens, the criterion of 10 or more per highpower microscope field has been adopted worldwide. Although this diagnostic criteria has also adopted that guideline, since there are inflammatory lesions or tumors other than AIP, which also meet this criteria, pathological findings are alone not sufficient for making a definite diagnosis.

3. Storiform fibrosis is a lesion comprised of inflammatory cell infiltration (lymphocytes, plasmacytes) and spindle-shaped cell hyperplasia, which presents complex cell arrangements characterized by the expression "storiform", and associated with differing degrees of fibrosis. The storiform most often appears in the pancreatic rim and peripancreatic fat tissues.

4. Obliterative phlebitis is a finding where lesions caused by the infiltration and fibrosis of lymphocytes and plasmacytes in inter-lobules and peripancreatic fat tissues extend into a vein to cause venous stenosis or occlusion.

Either a resected or biopsied pancreatic specimen may be used for the diagnosis. EUS-FNA cytological examination is extremely effective in differentiating AIP from malignant tumors, but is not effective in diagnosing AIP. EUS-FNA biopsy examination does not provide a definite diagnosis of AIP in most cases, since the amount of specimen is insufficient. EUS-core biopsy is reported to be effective in AIP diagnosis. Diagnosis of AIP using biopsied specimens requires caution, since pancreatic cancer also shows a large number of IgG4-positive plasmacytes in and around the pancreas in some cases, and pathological findings similar to LPSP in some isolated cases.

\section{[Notes] Type 2 AIP (IDCP)}

IDCP is a pancreatitis of unknown cause, which is characterized by the infiltration of neutrophils into the lumen or epithelium of the interlobular pancreatic duct. As in the case of LPSP, clinical differentiation from pancreatic cancer becomes an issue. Because of its similarity to LPSP in being associated with the infiltration and fibrosis of lymphocytes/plasmacytes around the pancreatic epithelium, IDCP was once thought to be in the same category as LPSP. Currently, IDCP cannot be diagnosed by images or clinical findings, but requires histopathological examinations. In addition, while resected or necropsied specimens of pancreas are large enough for a definite diagnosis, biopsied specimens are so small that a definite diagnosis is difficult in many cases. If typical pancreatic images of AIP are shown without abnormal hematological evidence, the disease could be either type 1 or type 2 . Some of type 2 AIP present clinical symptoms or image findings similar to those of pancreatic cancer, which makes it extremely difficult to differentiate type 2 AIP from pancreatic cancer.

V. Other organ involvement: OOI

1. Other organ involvement (OOI) observed in AIP refers to the IgG4 related lesions associated with type 1 .

2. Other organs reported to be affected include the central nervous system, lacrimal/salivary glands, thyroid glands, lungs, biliary duct, liver, gastrointestinal tracts, gallbladder, kidneys, prostate glands, retroperitoneum, and lymph nodes. In the lymph nodes and lacrimal glands, however, fibrosis is scarce; not all of these organs have established concepts of their lesions. If the following conditions are met, there may be a close relation with AIP, although no clear basis is available.

1) Investigations/reports of many cases show association with AIP.

2) Histopathological findings feature the infiltration and fibrosis of lymphocytes, obliterative phlebitis, and the infiltration of IgG4-positive plasmacytes into segmental lesions.

3) Steroid therapy is effective; or, the onset and offset of the effect synchronizes between pancreatic lesions and the lesions in question.

4) There are clear points that differentiate from diseases of each organ.

Diseases that satisfy the above conditions include sclerosing cholangitis, sclerosing dacryoadenitis/sialoadenitis 
(Mikulicz disease), retroperitoneal fibrosis, respiratory lesions, and tubulo-interstitial nephritis. Currently consensus is limited to sclerosing cholangitis, sclerosing dacryoadenitis/sialadenitis and retroperitoneal fibrosis.

3. Sclerosing cholangitis

1) The sclerosing cholangitis associated with AIP shows lesions over a wide area of the bile duct system; the stenosis of the lower bile duct caused by AIP must be differentiated from that caused by pancreatic cancer or cancer of the lower bile duct, and the stenosis of the intrahepatic and hilar bile ducts caused by AIP must be differentiated from that caused by primary sclerosing cholangitis (PSC) or bile duct cancer. It is necessary to make careful and comprehensive differentiation using not only the bile duct images but also endoscopic ultrasoundscopy (EUS), intraductal ultrasonography (IDUS), cytological and/or histological diagnosis, etc.

2) PSC is a different entity from the sclerosing cholangitis seen in AIP, because their responses to steroid therapy and prognoses are different. Findings characteristic to PSC are band-like strictures (e.g., short band-like strictures of 1-2 mm), a beaded appearance (e.g., alternating short strictures and dilatations), a pruned tree appearance (e.g., a reduced number of intrahepatic duct branches), and diverticulum-like outpouching.

3) It is controversial among specialists whether to include cases showing only lower bile duct stenosis within IgG4related sclerosing cholangitis, or to view them as part of the pancreatic lesions. The findings in bile duct lesions effective in diagnosing AIP are stenosis of the intrahepatic and hilar bile ducts and the sclerosing images or wall thickening of the upper and middle bile ducts.

4) Most of the pathological studies show a thickened bile duct and prominent transmural infiltration and fibrosis of lymphocytes and plasmacytes. Many IgG4-positive plasmacytes are observed in the lesions. The epithelium of the bile duct remains normal in most cases. Storiform fibrosis and obstructive phlebitis are also observed.

4. The IgG4 immunostaining of enlarged duodenal papillary biopsy specimens may be useful as a supporting diagnosis, although this enlargement is infrequent. An enlarged duodenal papillary is considered to be spread from lesions of the pancreatic head, and, therefore, is not in the scope of extra-pancreatic lesions (other organ involvement).

5. Sclerosing dacryoadenitis/sialoadenitis

1) Sclerosing dacryoadenitis/sialoadenitis associated with AIP shows no or slight (if any) symptoms of dry eye or dry mouth caused by decreased function of the lacrimal glands. Unlike Sjögren's Syndrome which is often associated with swollen parotid glands, sialoadenitis seen in AIP often demonstrates swollen submandibular glands and responds very well to steroid therapy. While most enlargement of the lacrimal and salivary glands is symmetrical, enlargement of the salivary glands is part of the enlarged submandibular, sublingual, or minor salivary glands. Dacryoadenitis/sialoadenitis in most cases test negative for anti SS-A antibody and anti SS-B antibody, which is different from Sjögren's Syndrome. The disease can be diagnosed based on the diagnostic criteria of the organs (diagnostic criteria for IgG4related Mikulicz disease, Japan Sjögren's Syndrome Study Group, 2008), however, if prominent infiltration of IgG4-positive plasmacytes is observed; it may be diagnosed by labial lip biopsy analysis.

2) Pathological findings show the disappearance of acinar cells in the lobule, prominent infiltration of lymphocytes and plasmacytes, formation of lymphoid follicles, and interlobular fibrosis. In some cases, the lobular structure may be destroyed, and prominent infiltration and fibrosis of lymphocytes and plasmacytes may form diffuse lesions. Many of the plasmacytes are IgG4 positive. Storiform fibrosis and obliterative phlebitis may be observed, although the incidence rate is lower compared with that of AIP.

6. Retroperitoneal fibrosis

1) Due to diffuse hyperplasmia and the inflammation of fibrous connective tissues on and around the retroperitoneum, abdominal CT/MRI images show soft shadows or mass around the abdominal aorta. This can cause ureteral obstruction, and subsequent hydrophrosis occasionally provide a clue for diagnosis. In some cases, the disease is associated with dilated lesions of the abdominal aorta and the condition known as inflammatory abdominal aortic aneurysm may be present; however, it is difficult to differentiate said aortic aneurysm from those caused by other etiologies.

2) Pathological study shows mass lesions formed by prominent infiltration and fibrosis of lymphocytes and plasmacytes. Many IgG4-positive plasmacytes are seen in the lesions. Storiform fibrosis and obliterative phlebitis are also seen very frequently.

\section{Option: effectiveness of steroid therapy}

Targets are the lesions for which image evaluation is possible; clinical conditions or hematological findings are not subject to effect evaluations. If no sufficient effect is seen within 2 weeks, reexamination is necessary. Effort should be made to take biopsies for pathological examination as much as possible, and facile diagnostic treatment with steroids should be strictly avoided. The administration of steroids may be effective in improving malignant lymphoma. 
VII. Endocrine and exocrine pancreatic functions

Typical AIP shows impaired exocrine pancreatic functions and diabetes. There are quite a few cases where steroid administration is effective in improving impaired endocrine and exocrine pancreatic functions

\section{Appendix 2: The Working Committee of the Japan Pancreas Society (JPS) and the Research Committee for Intractable Pancreatic Disease supported by the Ministry of Health, Labour and Welfare of Japan (RCIPD-MHLWJ):}

I. The professional committee for making clinical questions and statements

Chairperson: Kazuichi Okazaki (Department of Gastroenterology and Hepatology, Kansai Medical University)

Co-Chairpersons: Shigeyuki Kawa (Center for Health, Safety and Environmental Management, Shinshu University), and Terumi Kamisawa (Department of Internal Medicine, Tokyo Metropolitan Komagome Hospital)

Committee members:

Tetsuhide Ito (Department of Medicine and Bioregulatory Science, Graduate School of Medical Sciences, Kyushu University), Kazuo Inui (Department of Gastroenterology, Second Teaching Hospital, Fujita Health University), Hiroyuki Irie (Department of Radiology, Faculty of Medicine, Saga University), Takayoshi Nishino (Department of Gastroenterology, Tokyo Women's Medical University, Yachiyo Medical Center), Kenji Notohara (Department of Anatomic Pathology, Kurashiki Central Hospital), Keishi Kubo (Department of Internal Medicine, Shinshu University School of Medicine), Hirotaka Ohara (Department of Community-Based Medical Education, Nagoya City University Graduate School of Medical Sciences), Atsushi Irisawa (Department of Gastroenterology, Fukushima Medical University Aizu Medical Center), Yasunari Fujinaga (Department of Radiology, Shinshu University School of Medicine), Osamu Hasebe (Department of Gastroenterology, Nagano Municipal Hospital), Isao Nishimori (Nishimori Clinic), and Shigeki Tanaka (Department of Acupuncture and Moxibusion, Tokyo Ariake University of Medical and Health Sciences)

II. The expert panelist committee for rating statements by the modified Delphi method

Chairperson: Tooru Shimosegawa

Committee members: Kazuichi Okazaki, Shigeyuki Kawa, Terumi Kamisawa, Tetsuhide Ito, Kazuo Inui, Takayoshi Nishino, Hirotaka Ohara, Isao Nishimori, and Shigeki Tanaka
III. The Evaluating Committee

Chairperson: Masao Tanaka (Department of Surgery and Oncology, Kyushu University)

1. Committee members:

Toshimasa Nishiyama (Department of Public Health and Hygiene, Kansai Medical University), Koichi Suda (Department of Pathology, Tokyo-West Tokushukai Hospital), Keiko Shiratori (Department of Gastroenterology, Tokyo Women's Medical University), Kenji Notohara, Keishi Kubo, Hiroshi Yamamoto, Hirotaka Ohara, Atsushi Irisawa, Yasunari Fujinaga, Osamu Hasebe, and Shigeki Tanaka

2. Committee Members of the JPS for Autoimmune Pancreatitis:

Kazushige Uchida (Department of Gastroenterology and Hepatology, Kansai Medical University), Atsushi Kanno (Division of Gastroenterology, Tohoku University Graduate School of Medicine), Kensuke Kubota (Department of Gastroenterology, Yokohama City Unicersity), Shigeru Ko (Department of Systems Medicine, Keio University), Junichi Sakagami (Department of Gastroenterology and Hepatology, Kyoto Prefectural University of Medicine), Kyoko Shimizu (Department of Gastroenterology, Tokyo Women's Medical University), Masanori Sugiyama (Department of Surgery, Kyorin University), Minoru Tada (Department of Gastroenterology, University of Tokyo), Takahiro Nakazawa (Department of Gastroenterology and Metabolism, Nagoya City University), Hirokazu Nishino (Department of Gastroenterology and Hepatology, Jikei University School of Medicine), Hideaki Hamano (Medical Informatics Division and Department of Internal Medicine, Gastroenterology, Shinshu University Hospital), Yoshiki Hirooka (Department of Endoscopy, Nagoya University Hospital), Kenji Hirano (Department of Gastroenterology, University of Tokyo), Atsushi Masamune (Division of Gastroenterology, Tohoku University Graduate School of Medicine), Atsuhiro Masuda (Divison of Gastroenterology, Department of Internal Medicine, Kobe University Graduate School of Medicine), Nobumasa Mizuno (Department of Gastroenterology, Aichi Cancer Center Hospital), Koji Yamaguchi (Department of Surgery 1, University of Occupational and Environmental Health), and Hitoshi Yoshida (Division of Gastroenterology, Department of Medicine, Showa University School of Medicine)

\section{References}

1. Yoshida K, Toki F, Takeuchi T, et al. Chronic pancreatitis caused by an autoimmune abnormality. Proposal of the concept of autoimmune pancreatitis. Dig Dis Sci. 1995;40:1561-8. 
2. Hamano H, Kawa S, Horiuchi A, et al. High serum $\operatorname{IgG} 4$ concentrations in patients with sclerosing pancreatitis. N Engl J Med. 2001;344:732-8.

3. Japan Pancreas Society.Clinical diagnostic criteria for autoimmune pancreatitis.Suizo 2002; 17:585-7.

4. Okazaki K, Kawa S, Kamisawa T, et al. Clinical diagnostic criteria of autoimmune pancreatitis: revised proposal. J Gastroenterol. 2006;41:626-31.

5. Members of the Autoimmune Pancreatitis Diagnostic Criteria Committee, the Research Committee of Intractable Diseases of the Pancreas supported by the Japanese Ministry of Health, Labour and Welfare, and Members of the Autoimmune Pancreatitis Diagnostic Criteria Committee, the Japan Pancreas Sociey. Clinical diagnostic criteria of autoimmune pancreatitis 2006. Suizo. 2006;21:395-7. (In Japanese with English abstract).

6. Okazaki K, Kawa S, Kamisawa T, et al. Japanese consensus guidelines for management of autoimmune pancreatitis: I. Concept and diagnosis of autoimmune pancreatitis. J Gastroenterol. 2010;45:249-65.

7. Kawa S, Okazaki K, Kamisawa T, et al. Japanese consensus guidelines for management of autoimmune pancreatitis: II. Extrapancreatic lesions, differential diagnosis. J Gastroenterol. 2010;45(4):355-69.

8. Kamisawa T, Okazaki K, Kawa $\mathrm{S}$, et al. Japanese consensus guidelines for management of autoimmune pancreatitis: III. Treatment and prognosis of AIP. J Gastroenterol. 2010;45(5):471-7.

9. Shimosegawa T, Chari ST, Frulloni L, et al. International Consensus Diagnostic Criteria for Autoimmune Pancreatitis: Guidelines of the International Association of Pancreatology. Pancreas. 2011;40:352-8.

10. The Japan Pancreas Society, the Ministry of Health and Welfare Investigation Research Team for Intractable Pancreatic Disease. Suizo 2012; 27:17-25. (In Japanese with English abstract).

11. Shimosegawa T, et al. The amendment of the Clinical Diagnostic Criteria in Japan (JPS2011) in response to the proposal of the International Consensus of Diagnostic Criteria (ICDC) for autoimmune pancreatitis. Pancreas. 2012;41:1341-2.

12. Fitch K, Bernstein SJ, Aguilar MD, et al. The RAND/UCLA appropriateness method user's manual. Santa Monica: RAND; 2001.

13. U.S. Preventive Services Task Force http://www.ahrq.gov/clinic/ 3rduspstf/ratings.htm.

14. Kamisawa T, Funata N, Hayashi Y, et al. A new clinicopathological entity of IgG4-related autoimmune disease. J Gastroenterol. 2003;38:982-4.

15. Yamamoto M, Takahashi H, Ohara M, et al. A new conceptualization for Mikulicz's disease as an IgG4-related plasmacytic disease. Mod Rheumatol. 2006;16:335-40.

16. Masaki Y, Dong L, Kurose N, et al. Proposal for a new clinical entity, IgG4-positive multi-organ lymphoproliferative syndrome: analysis of 64 cases of IgG4-related disorders. Ann Rheum Dis. 2009;68:1310-5.

17. Umehara H, Okazaki K, Masaki Y, et al. A novel clinical entity, IgG4-related disease (IgG4RD): general concept and details. Mod Rheumatol. 2012;22:1-14

18. Stone JH, Khosroshahi A, Deshpande V, et al. IgG4-Related disease: recommendations for the nomenclature of this condition and its individual organ system manifestations. Arthritis Rheum. 2012;64:3061-7.

19. Okazaki K, Uchida K, Koyabu M, et al. Recent advances in the concept and diagnosis of autoimmune pancreatitis and IgG4related disease. J Gastroenterol. 2011;46:277-88.

20. Okazaki K, Uchida K, Ikeura $T$, et al. Current concept and diagnosis of IgG4-related disease in the hepato-bilio-pancreatic system. J Gastroenterol. 2013;48:303-14.
21. Kawaguchi K, Koike M, Tsuruta K, et al. Lymphoplasmacytic sclerosing pancreatitis with cholangitis: a variant primary sclerosing cholangitis extensively involving pancreas. Hum Pahtol. 1991;22:387-95.

22. Notohara K, Burgart LJ, Yadav D, et al. Idiopathic chronic pancreatitis with periductal lymphoplasmacytic infiltration: clinicopathologic features of 35 cases. Am J Surg Pathol. 2003;27:1119-27.

23. Zamboni G, Luttges J, Capelli P, et al. Histopathological features of diagnostic and clinical relevance in autoimmune pancreatitis: a study on 53 resection specimens and 9 biopsy specimens. Virchows Arch. 2004;445:552-63.

24. Klöppel G, Detlefsen S, Chari ST, et al. Autoimmune pancreatitis: the clinicopathological characteristics of the subtype with granulocytic epithelial lesions. J Gastroenterol. 2010;45:787-93.

25. Kamisawa T, Okamoto A, Funata N. Clinicopathological features of autoimmune pancreatitis in relation to elevation of serum IgG4. Pancreas. 2005;31:28-31.

26. Kamisawa T, Wakabayashi T, Sawabu N. Autoimmune pancreatitis in young patients. J Clin Gastroenterol. 2006;40:847-50.

27. Kawa S, Hamano H. Clinical features of autoimmune pancreatitis. J Gastroenterol. 2007;42(Suppl 18):9-14.

28. Uchida K, Okazaki K, Konishi Y, et al. Clinical analysis of autoimmune-related pancreatitis. Am J Gastroenterol. 2000;95: 2788-94.

29. Nagata M, Yoshino J, Inui K, et al. A case of autoimmune pancreatitis following acute pancreatitis associated with septicemia. Suizo. 2003;18:215-20.

30. Sumida A, Kanemasa K, Tachibana S, et al. A case of autoimmune pancreatitis occurring during intravesical Bacillus Calmette Guerin immunotherapy for ureteral cancer. Jpn J Gastroenterol. 2003;100:1328-32.

31. Kanno A, Nishimori I, Masamune A, et al. Nationwide epidemiological survey of autoimmune pancreatitis in Japan. Pancreas. 2012;41(6):835-9.

32. Okazaki K, Uchida K, Ohana M, et al. Autoimmune-related pancreatitis is associated with autoantibodies and a Th1/Th2-type cellular immune response. Gastroenterology. 2000;118:573-81.

33. Okazaki K. Autoimmune pancreatitis: etiology, pathogenesis, clinical findings and treatment. The Japanese experience. JOP. 2005;6(1 Suppl):89-96.

34. Shigeyuki K, Hideaki H. Serological markers for the diagnosis of autoimmune pancreatitis. Suizo. 2007;22:641-5 (In Japanese with English abstract).

35. Nishimori I, Tamakoshi A, Kawa S, et al. Influence of steroid therapy on the course of diabetes mellitus in patients with autoimmune pancreatitis: findings from a nationwide survey in Japan. Pancreas. 2006;32:244-8.

36. Zen Y, Fujii T, Harada K, et al. Th2 and regulatory immune reactions are increased in immunoglobin G4-related sclerosing pancreatitis and cholangitis. Hepatology. 2007;45:1538-46.

37. Tanaka A, Moriyama M, Nakashima $\mathrm{H}$, et al. Th2 and regulatory immune reactions contribute to IgG4 production and the initiation of Mikulicz disease. Arthritis Rheum. 2012;64:254-63.

38. Kamisawa T, Egawa N, Inokuma S, et al. Pancreatic endocrine and exocrine function and salivary gland function in autoimmune pancreatitis before and after steroid therapy. Pancreas. 2003;27:235-8.

39. Nishino $T$, Toki $F$, Oyama $H$, et al. Long-term outcome of autoimmune pancreatitis after oral prednisolone therapy. Intern Med. 2006;45:497-501.

40. Ito $\mathrm{T}$, Nishimori I, Inoue $\mathrm{N}$, et al. Treatment for autoimmune pancreatitis: consensus on the treatment for patients with autoimmune pancreatitis in Japan. J Gastroenterol. 2007;42(Suppl 18):50-8. 
41. Tanaka S, Kobayashi T, Nakanishi K, et al. Corticosteroidresponsive diabetes mellitus associated with autoimmune pancreatitis. Lancet. 2000;356:910-1.

42. Ko SB, Mizuno N, Yatabe Y, et al. Corticosteroids correct aberrant CFTR localization in the duct and regenerate acinar cells in autoimmune pancreatitis. Gastroenterology. 2010;138(5):1988-96.

43. Yoda Y, Kobayashi K, Enomoto N. Study of category classification in abdominal ultrasonographic mass screening. J Gastroenterol Mass Surv. 2006;44:12-20.

44. Irie H, Ito T. US, CT and MRI findings of autoimmune pancreatitis based on "Clinical diagnostic criteria of autoimmune pancreatitis 2006". Suizo. 2007;22:629-33 (In Japanese with English abstract).

45. Kamisawa $\mathrm{T}$, Egawa $\mathrm{N}$, Nakajima $\mathrm{H}$, et al. Comparison of radiological and histological findings in autoimmune pancreatitis. Hepatogastroenterol. 2006;53(72):953-6.

46. Muraki T, Ozaki Y, Hamano H, et al. Ultrasonographic diagnosis of autoimmune pancreatitis. Biliary Tract Pancreas. 2005;26:711-6.

47. Honjo H, Takeuchi K, Nagashima N, et al. Biliary duct lesions associated with autoimmune pancreatitis. Biliary Tract Pancreas. 2001;22:581-7.

48. Hyodo N, Hyodo T. Ultrasonographic evaluation in patients with autoimmune-related pancreatitis. J Gastroenterol. 2003;38(12):1155-61.

49. Hasebe O, Arakura N, Imai Y, et al. The findings of ERC and IDUS in autoimmune pancreatitis. $\mathrm{J}$ Gastroenterol Imaging. 2002;4:41-8.

50. Nagase M, Furuse J, Ishii $\mathrm{H}$, et al. Evaluation of contrast enhancement patterns in pancreatic tumors by coded harmonic sonographic imaging with a microbubble contrast agent. J Ultrasound Med. 2003;22(8):789-95.

51. Numata K, Ozawa Y, Kobayashi N, et al. Contrast-enhanced sonography of autoimmune pancreatitis: comparison with pathologic findings. J Ultrasound Med. 2004;23(2):199-206.

52. Miyoshi H, Inui K, Yoshino J, et al. Usefulness of contrastenhanced ultrasonography in autoimmune pancreatitis. Suizo. 2010;25:117-24 (In Japanese with English abstract).

53. Irie $\mathrm{H}, \mathrm{Honda} \mathrm{H}, \mathrm{Baba} \mathrm{S}$, et al. Autoimmune pancreatitis: $\mathrm{CT}$ and MR characteristics. AJR Am J Roentgenol. 1998;170:1323-7.

54. Sahani DV, Kalva SP, Farrell J, et al. Autoimmune pancreatitis: imaging features. Radiology. 2004;233:345-52.

55. Suzuki K, Itoh S, Nagasaka T, et al. CT findings in autoimmune pancreatitis: assessment using multiphase contrast-enhanced multisection CT. Clin Radiol. 2010;65(9):735-43.

56. Hirota M, Tsuda M, Tsuji Y, et al. Perfusion computed tomography findings of autoimmune pancreatitis. Pancreas. 2011;40(8):1295-301.

57. Ishigami K, Tajima T, Nishie A, et al. MRI findings of pancreatic lymphoma and autoimmune pancreatitis: a comparative study. Eur J Radiol. 2010;74(3):e22-8.

58. Kamisawa T, Takuma K, Anjiki $\mathrm{H}$, et al. Differentiation of autoimmune pancreatitis from pancreatic cancer by diffusionweighted MRI. Am J Gastroenterol. 2010;105(8):1870-5.

59. Hur BY, Lee JM, Lee JE, et al. Magnetic resonance imaging findings of the mass-forming type of autoimmune pancreatitis: comparison with pancreatic adenocarcinoma. J Magn Reson Imaging. 2012;36(1):188-97.

60. Muhi A, Ichikawa T, Motosugi U, et al. Mass-forming autoimmune pancreatitis and pancreatic carcinoma: differential diagnosis on the basis of computed tomography and magnetic resonance cholangiopancreatography, and diffusion-weighted imaging findings. J Magn Reson Imaging. 2012;35(4):827-36.

61. Horiuchi A, Kaneko T, Yamamura N, et al. Autoimmune chronic pancreatitis simulating pancreatic lymphoma. Am J Gastroenterol. 1996;91:2607-9.
62. Saegusa H, Momose M, Kawa S, et al. Hilar and pancreatic gallium-67 accumulation is characteristic feature of autoimmune pancreatitis. Pancreas. 2003;27:20-5.

63. Higashi T, Saga T, Nakamoto Y, et al. Diagnosis of pancreatic cancer using fluorine-18 fluorodeoxyglucose positron emission tomography (FDG PET) -usefulness and limitations in "clinical reality". Ann Nucl Med. 2003;17:261-79.

64. Nakamoto Y, Sakahara H, Higashi T, et al. Autoimmune pancreatitis with F-18 fluoro-2-deoxy-D-glucose PET findings. Clin Nucl Med. 1999;24:778-80.

65. Nakamoto Y, Saga T, Ishimori T, et al. FDG-PET of autoimmune-related pancreatitis: preliminary results. Eur J Nucl Med. 2000;27:1835-8

66. Nakajo M, Jinnouchi S, Fukukura Y, et al. The efficacy of wholebody FDG-PET or PET/CT for autoimmune pancreatitis and associated extrapancreatic autoimmune lesions. Eur J Nucl Med Mol Imaging. 2007;34:2088-95.

67. Ozaki Y, Oguchi K, Hamano H, et al. Differentiation of autoimmune pancreatitis from suspected pancreatic cancer by fluorine-18 fluorodeoxyglucose positron emission tomography. J Gastroenterol. 2008;43:144-51.

68. Sato M, Okumura T, Shioyama Y, et al. Extrapancreatic F-18 FDG accumulation in autoimmune pancreatitis. Ann Nucl Med. 2008;22:215-9.

69. Nishimori I, Kohsaki T, Onishi S, et al. IgG4-related autoimmune prostatitis: two cases with or without autoimmune pancreatitis. Intern Med. 2007;46:1983-9.

70. Shigekawa M, Yamao K, Sawaki A, et al. Is (18)F-fluorodeoxyglucose positron emission tomography meaningful for estimating the efficacy of corticosteroid therapy in patients with autoimmune pancreatitis? J Hepatobiliary Pancreat Surg. 2009;17:269-74.

71. Toki F, Kozu T, Oi I. An usual type of chronic pancreatitis showing diffuse narrowing of the entire main pancreatic duct on ERCP. A report of four cases. Endoscopy. 1992;24:640.

72. Wakabayshi T, Kawamura Y, Satomura Y, et al. Clinical and imaging features of autoimmune pancreatitis with focal pancreatic swelling or mass formation; comparison with so-called tumor-forming pancreatitis and pancreatic cancer. Am J Gastroenterol. 2003;98:2679-87.

73. Nakazawa T, Ohara H, Sano H, et al. Difficulty in diagnosing autoimmune pancreatitis. Gastrointest Endosc. 2007;65:99-108.

74. Nishino T, Oyama H, Toki F, et al. Differentiation between autoimmune pancreatitis and pancreatic carcinoma based on endoscopic retrograde cholangiopancreatography findings. J Gastroenterol. 2010;45:988-96.

75. Kamisawa T, Imai M, Yui Chen P, et al. Strategy for differentiating autoimmune pancreatitis from pancreatic cancer. Pancreas 2008;37:e62-7.

76. Kamisawa $\mathrm{T}, \mathrm{Tu} \mathrm{Y}$, Egawa $\mathrm{N}$, et al. Involvement of pancreatic and bile ducts in autoimmune pancreatitis. World J Gastroenterol. 2006;12:612-4.

77. Ito T, Nakano I, Koyanagi S, et al. Autoimmune pancreatitis as a new clinical entity; three cases of autoimmune pancreatitis with effective steroid therapy. Dig Dis Sci. 1997;42:1458-68.

78. Horiuchi A, Kawa S, Hamano H, et al. ERCP features in 27 patients with autoimmune pancreatitis. Gastrointest Endosc. 2002;4:494-9.

79. Nishino T, Toki F, Oyama H, et al. Biliary tract involvement in autoimmune pancreatitis. Pancreas. 2005;30:76-82.

80. Nakazawa T, Ohara H, Sano H, et al. Cholangiography can discriminate sclerosing cholangitis with autoimmune pancreatitis from primary sclerosing cholangitis. Gastrointest Endosc. 2004;60:937-44.

81. Hirano K, Shiratori Y, Komatsu Y, et al. Involvement of the biliary system in autoimmune pancreatitis: a follow-up study. Clin Gastroenterol Hepatol. 2003;1:453-64. 
82. Ectors N, Maillet B, Aerts R, et al. Non-alcoholic duct destructive chronic pancreatitis. Gut. 1997;41:263-8.

83. Suda K, Takase M, Fukumura Y, et al. Histopathologic characteristics of autoimmune pancreatitis based on comparison with chronic pancreatitis. Pancreas. 2005;30:355-8.

84. Suda K, Nishimori I, Takase M, et al. Autoimmune pancreatitis can be classified into early and advanced stages. Pancreas. 2006;33:345-50.

85. Deshpande V, Chicano S, Finkelberg D, et al. Autoimmune pancreatitis: a systemic immune complex mediated disease. Am J Surg Pathol. 2006;30:1537-45.

86. Kojima M, Sipos B, Klapper W, et al. Autoimmune pancreatitis: frequency, IgG4 expression, and clonality of T and B cells. Am J Surg Pathol. 2007;31:521-8.

87. Aoki $\mathrm{S}$, Nakazawa $\mathrm{T}$, Ohara $\mathrm{H}$, et al. Immunohistochemical study of autoimmune pancreatitis using anti-IgG4 antibody and patients' sera. Histopathology. 2005;47:147-58.

88. Zhang L, Notohara K, Levy MJ, et al. IgG4-positive plasma cell infiltration in the diagnosis of autoimmune pancreatitis. Mod Pathol. 2007;20:23-8.

89. Deshpande V, Zen Y, Chan JK, et al. Consensus statement of the pathology of IgG4-related disease. Mod Pathol. 2012;25(9):1181-92.

90. Mizuno N, Bhatia V, Hosoda W, et al. Histological diagnosis of autoimmune pancreatitis using EUS-guided trucut biopsy: a comparison study with EUS-FNA. J Gastroenterol. 2009;44: $742-50$.

91. Levy MJ, Reddy RP, Wiersema MJ, et al. EUS-guided trucut biopsy in establishing autoimmune pancreatitis as the cause of obstructive jaundice. Gastrointest Endosc. 2005;61:467-72.
92. Imai K, Matsubayashi H, Fukutomi A, et al. Endoscopic ultrasonography-guided fine needle aspiration biopsy using 22-gauge needle in diagnosis of autoimmune pancreatitis. Dig Liver Dis. 2011;43:869-74.

93. Kanno A, Ishida K, Hamada $S$, et al. Diagnosis of autoimmune pancreatitis by EUS-FNA by using a 22-gauge needle based on the International Consensus Diagnostic Criteria. Gastrointest Endosc. 2012;76:594-602.

94. Chari ST, Smyrk TC, Levy MJ, et al. Diagnosis of autoimmune pancreatitis: the Mayo Clinic experience. Clin Gastroenterol Hepatol. 2006;4:1010-6.

95. Kim K, Kim MW, Kim JC, et al. Diagnostic criteria for autoimmune pancreatitis revisited. World $\mathrm{J}$ Gastroenterol. 2006;12:2487-96.

96. Otsuki M, Chung JB, Okazaki K, et al. Asian diagnostic criteria for autoimmune pancreatitis: consensus of the Japan-Korea Symposium on Autoimmune Pancreatitis. J Gastroenterol. 2008;43:403-8.

97. Kamisawa T, Tu Y, Nakajima $\mathrm{H}$, et al. Usefulness of biopsying the major duodenal papilla to diagnose autoimmune pancreatitis: a prospective study using IgG4-immunostaining. World J Gastroenterol. 2006;12:2031-3.

98. Kubota K, Iida H, Fujisawa T, et al. Clinical significance of swollen duodenal papilla in autoimmune pancreatitis. Pancreas. 2007;35(4):e51-60. 